\title{
Cells use molecular working memory to navigate in changing chemoattractant fields
}

\author{
Akhilesh Nandan ${ }^{1,2, \dagger}$, Abhishek Das ${ }^{1,2, \dagger}$, Robert Lott $^{2}$ and Aneta Koseska $a^{1,2, *}$ \\ ${ }^{1}$ Department of Systemic Cell Biology, Max Planck Institute of Molecular Physiology, \\ Otto-Hahn-Str.11, 44227 Dortmund, Germany \\ 2 Current address: Cellular Computations and Learning, \\ Center of Advanced European Studies and Research (Caesar), Ludwig-Erhard-Allee 2, 53175 Bonn, Germany \\ $\dagger$ These authors contributed equally. \\ *To whom correspondence should be addressed; E-mail: aneta.koseska@caesar.de
}

\section{Abstract}

In order to migrate over large distances, cells within tissues and organisms rely on sensing 2 local gradient cues. These cues however are multifarious, irregular or conflicting, changing 3 both in time and space. Here we find that single cells utilize a molecular mechanism akin to 4 a working memory, to generate persistent directional migration when signals are disrupted by 5 temporally memorizing their position, while still remaining adaptive to spatial and temporal 6 changes of the signal source. Using dynamical systems theory, we derive that these information 7 processing capabilities are inherent for protein networks whose dynamics is maintained away 8 from steady state through organization at criticality. We demonstrate experimentally using the 9 Epidermal growth factor receptor (EGFR) signaling network, that the memory is maintained 10 in the prolonged receptor's activity via a slow-escaping remnant, a dynamical "ghost" of the 11 
attractor of the polarized signaling state, that further results in memory in migration. As this 12 state is metastable, it also enables continuous adaptation of the migration direction when the $\quad 13$ signals vary in space and time. We therefore show that cells implement real-time computations 14 without stable-states to navigate in changing chemoattractant fields by memorizing position of 15 disrupted signals while maintaining sensitivity to novel chemical cues.

\section{Introduction}

Directed chemotactic behavior relies on generating polarized signaling activity at the plasma 18 membrane of the cell that is translated to an elongated cell shape in the direction of the signal. 19 Cells maintain the acquired orientation longer than the duration of the recently encountered 20 signal in order to avoid immediate switching to random migration when signals are temporarily $\quad 21$ disrupted or noisy, while simultaneously remaining sensitive and are able to adapt the migration 22 direction based on the changes in the environment (Parent and Devreotes, 1999; Foxman et al., 23 1999; Ridley et al., 2003). Thus, cells as diverse as social amoeba, neutrophils, leukocytes, fi- 24 broblasts and nerve cells, not only respond to dynamic gradients, but also integrate and resolve $\quad 25$ competing spatial signals or prioritize newly encountering attractants, likely by memorizing 26 their recent environment (Jilkine and Edelstein-Keshet, 2011; Skoge et al., 2014; Albrecht and ${ }_{27}$ Petty, 1998). Numerous models of chemotactic responses based on positive feedbacks, inco- 28 herent feed-forward, excitable or Turing-like networks have been proposed, accounting either $\quad{ }_{29}$ for sensing non-stationary stimuli or for long-term maintenance of polarized signaling activity, 30 but not both (Levchenko and Iglesias, 2002; Levine et al., 2002; Mori et al., 2008; Goryachev 31 and Pokhilko, 2008; Beta et al., 2008; Xiong et al., 2010; Trong et al., 2014; Halatek and Frey, 32 2018). These models rely on computations with stable states, where switching from the at- ${ }_{33}$ tractor of basal- to the attractor of polarized-signaling activity enables noise-robust sensing, 34 or establishing a long-term memory of previous signal localization. However, they are less 35 
suited for real-time computation of signals that vary in time and space, since the stable attrac- ${ }_{36}$ tors completely hinder or at least significantly delay the responsiveness to newly encountered 37 signals (Stanoev et al., 2020). Thus, the mechanism that underlies robust cellular navigation in 38 changing chemical fields has remained unknown.

Here we set out to identify how cells satisfy these two general, but seemingly opposed 40 demands: maintaining temporal memory in directional migration through a prolonged polarized ${ }^{41}$ state beyond the chemotactic signal duration, while still being able to quickly reset and re-adapt 42 upon novel sensory cues. Using a mathematical model of EGFR network signaling dynamics, 43 we predict and demonstrate experimentally in epithelial cells that these competing demands 44 are uniquely fulfilled for network's organization at criticality. Beyond this specific biological 45 implementation, we present a generic dynamical mechanism that addresses how cells compare 46 and integrate chemical cues over time and space in order to generate robust responses in a 47 history-dependent manner. 


\section{Results}

\section{Dynamical basis of navigation in non-stationary environ- 50 ments

We conjectured that operating in changing environments likely relies on computations with 52 metastable states rather than stable attractors, to allow both for transient stability of the polar- ${ }_{53}$ ized signaling state when signals are disrupted or noisy, as well as its rapid adaptation when 54 the signals vary in space and time. Our hypothesis is that this can be achieved if biochemical 55 systems are maintained away from steady state. We therefore approached the problem using 56 the abstract language of dynamical systems theory, where the characteristics of any process 57 directly follow from the type of dynamical transitions, called bifurcations, through which they 58 emerge (Strogatz, 2018). In our previous work we identified that when a saddle-node bifurca- $\quad 59$ tion $(S N)$ and thereby a steady-state is lost in a dynamical transition, i.e. upon signal removal, $\quad 60$ a remnant or a dynamical "ghost" of the stable attractor serves as a mechanism for sensing time- 61 varying growth factors in biochemical receptor networks (Stanoev et al., 2018; Stanoev et al., 62 2020). Necessary for manifestation of the "ghost" state is organization at criticality, which in the 63 networks we previously examined was determined by the concentration of receptors on the cell 64 membrane. Moreover, the "ghost" state is dynamically metastable and transiently maintains the 65 system in the vicinity of the steady state. Thus, a transient memory of previously present stim- 66 uli is generated, which enables integration of information contained in the temporal signals. $\quad 67$ Navigation in changing environments through directed migration however, must additionally 68 rely on a polarized representation of the directional signal, requiring a reliable mechanism for 69 signal-induced transition from a non-polarized symmetric to an asymmetric polarized recep- 70 tor signaling state and subsequently polarized cell shape. From a dynamical systems point of 71 view, a pitchfork bifurcation $(P B)$ can satisfy the condition for robust cell polarization, since 72 
by definition a $P B$ characterizes a transition from a homogeneous to an inhomogeneous steady $\quad 73$ state (Koseska et al., 2013; Strogatz, 2018). We thus hypothesized that organization at criti- 74 cality - in the vicinity of a $S N_{P B}$ through which a sub-critical $P B$ is stabilized (grey shaded 75 area in Figure 1A), the dynamical characteristics of both bifurcations, signal integration through 76 dynamic memory and cell polarization, will be uniquely manifested to render a minimal mech- 77 anism for responsiveness in changing environments.

We described this conjecture mathematically for a general reaction-diffusion model repre- 79 senting the signaling activity on the plasma membrane of a cell, $\frac{\partial \mathbf{U}(\mathbf{x}, \mathbf{t})}{\partial t}=\mathbf{F}(\mathbf{U})+\mathbf{D} \nabla^{2} \mathbf{U}(\mathbf{x}, \mathbf{t}), \quad 80$ with $\mathbf{U}$ being the vector of local densities of active signaling components, $\mathbf{D}$ - diffusion con- ${ }_{81}$ stants and $\mathbf{F}$ accounting for all chemical reactions. Our analysis shows that a $P B$ exists if, for a 82 spatial perturbation of the symmetric steady state $\left(\mathbf{U}_{\mathbf{s}}\right)$ of the form $\mathbf{U}(\mathbf{x}, t)=\mathbf{U}_{\mathbf{s}}+\delta \mathbf{U}(\mathbf{x}) e^{\lambda t}, \quad{ }^{83}$ the conditions $\delta \mathbf{U}(-\mathbf{x})=-\delta \mathbf{U}(\mathbf{x})$ and the $\operatorname{limit}_{\lim _{\lambda \rightarrow 0}} F_{\lambda}=\operatorname{det}(J)=0$ are simultaneously 84 fulfilled (Supplementary information). This implies that the linearized system has zero-crossing 85 eigenvalues $(\lambda)$ associated with the odd mode of the perturbation (Paquin-Lefebvre et al., 2020). $\quad 86$ To probe the sub-critical transition and therefore the necessary organization at criticality, a re- $\quad{ }_{87}$ duced description in terms of an asymptotic expansion of the amplitude of the polarized state 88 $(\phi)$ must yield the Landau equation $\frac{d \phi}{d t}=c_{1} \phi+c_{2} \phi^{3}-c_{3} \phi^{5}$, guaranteeing the existence of $\quad 89$ $S N_{P B}$ (see Supplementary information for derivation).

These abstract dynamical transitions can be realized in receptor tyrosine kinase signaling 91 networks with different topologies and are best analyzed using computational models, whose 92 predictions are then tested in quantitative experiments on living cells. To exemplify the above 93 mentioned principle, we use the well-characterized Epidermal growth factor receptor (EGFR) 94 sensing network (Reynolds et al., 2003; Baumdick et al., 2015; Stanoev et al., 2018). It con- 95 stitutes of double negative and negative feedback interactions of the receptor, EGFR $\left(E_{p}\right)$ with 96 two enzymes, the phosphatases PTPRG $\left(P_{R G}\right)$ and PTPN2 $\left(P_{N 2}\right.$; Figure 1B, Figure S1A), re- ${ }_{97}$ 
A

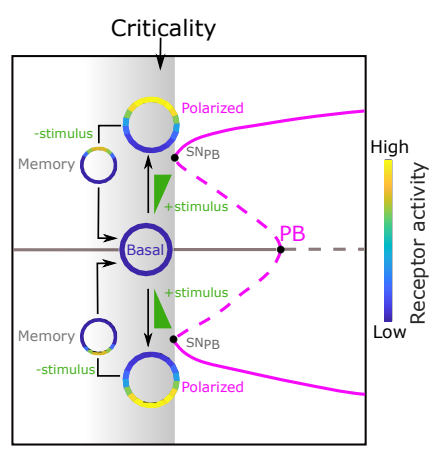

D
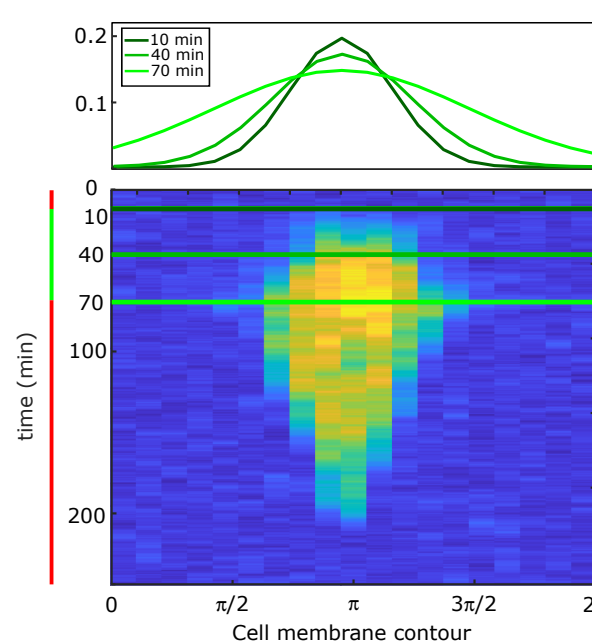

G

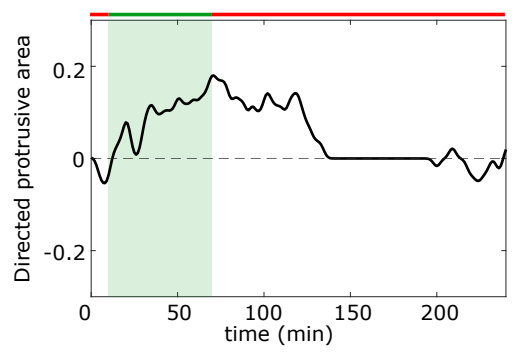

B

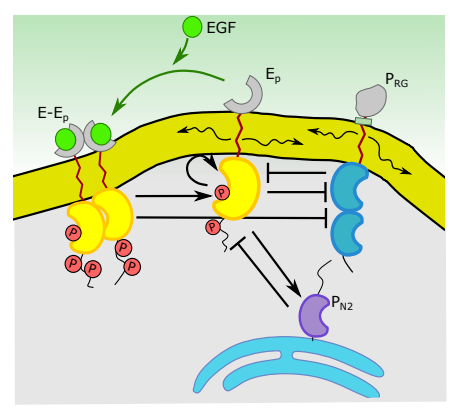

$\mathrm{E}$

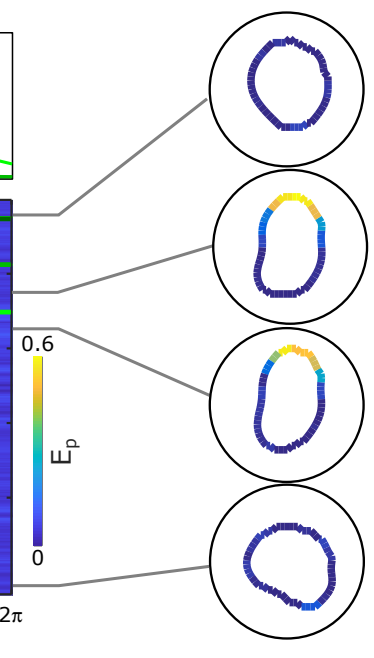

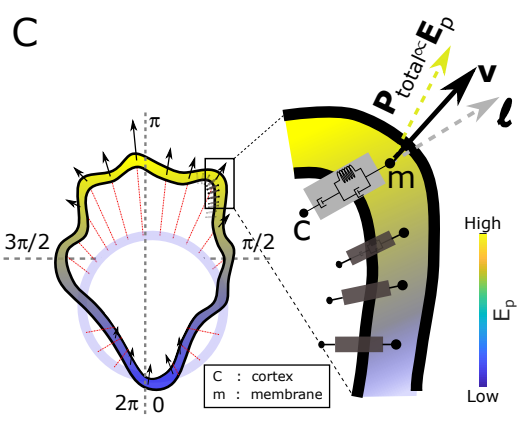

$\mathrm{F}$
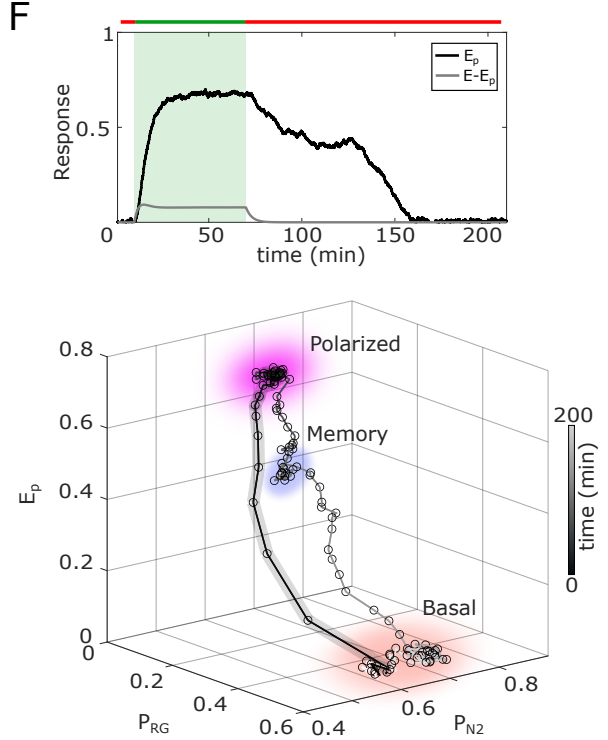

$\mathrm{H}$
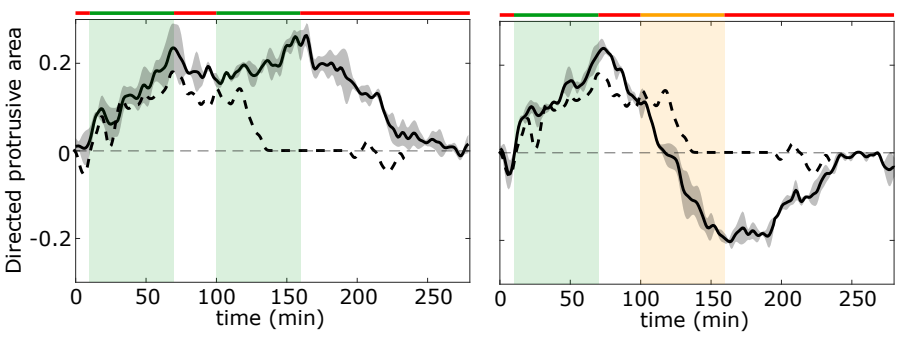

Figure 1. Organization at criticality enables sensing changing spatial-temporal signals. A, Dynamical mechanism: critical organization before sub-critical pitchfork bifurcation $(P B$, grey shaded area). Stable/unstable steady states (solid/dashed lines): basal (homogeneous, grey) and polarized (inhomogeneous, magenta) receptor activity; stimulus induced transitions between states: arrow lines. $S N_{P B}$ : saddle-node bifurcation through which $P B$ is stabilized. 
B, Scheme of the EGFR-PTP interaction network. Ligandless EGFR $\left(E_{p}\right)$ interacts with PT$\operatorname{PRG}\left(P_{R G}\right)$ and PTPN2 $\left(P_{N 2}\right)$. Liganded EGFR $\left(E-E_{p}\right)$ promotes autocatalysis of $E_{p}$. Causal links: solid black lines; curved arrow lines: diffusion. See also Figure S1A. C, Signal-induced shape-changes during cell polarization. Arrows: local edge velocity direction. Zoom: Viscoelastic model of the cell - parallel connection of an elastic and a viscous element. $\mathbf{P}_{\text {total }}$ : total pressure; v: local membrane velocity; l: viscoelastic state. Bold letters: vectors. Cell membrane contour: $[0,2 \pi]$. D, Top: In silico evolution of spatial EGF distribution. Bottom: Kymograph of $E_{p}$ for organization at criticality from reaction-diffusion simulations of the network in $(\mathbf{B})$. E, Corresponding exemplary cell shapes with color coded $E_{p}$, obtained with the model in (C). F, Top: Temporal profiles $E_{p}$ (black) and $E-E_{p}$ (grey). Green shaded area: EGF gradient presence. Bottom: State-space trajectory of the system with denoted trapping state-space areas (colored). See also movie S1. Thick/thin line: signal presence/absence. G, Quantification of in silico cell morphological changes from the example in $\mathbf{E}$. $\mathbf{H}$, Left: same as in $\mathbf{G}$, only when stimulated with two consecutive dynamic gradients from same direction. Second gradient within the memory phase of the first. Right: the second gradient (orange) has opposite localization. Mean \pm s.d. from $n=3$. See also Figure S1C,D. Dashed line: curve from G. Parameters: Supplementary information. In (D-H), green(orange)/red lines: stimulus presence/absence. See also Figure S1.

spectively. $E_{p}$ and $P_{R G}$ laterally diffuse on the membrane and inhibit each-other's activities 98 (see Supplementary information for the molecular details of the network). These molecular 99 interactions can be mathematically described using mass action kinetics (Eqs.(6) in Supple- 100 mentary information), and a weakly nonlinear analysis (Becherer et al., 2009) shows that the 101 EGFR signaling dynamics undergoes a symmetry-breaking transition as outlined above (proof 102 in Supplementary information, Figure S1B). Contrary to a bistable system, where the polarized ${ }_{103}$ signaling state would be manifested by two steady states, for e.g. high and low protein phos- ${ }_{104}$ phorylation in the front and back of the cell respectively (Beta et al., 2008), the inhomogeneous 105 steady state generated via a $P B$ is a single attractor defined as a combination of the front and 106 back activity states. This profiles $P B$ as a robust mechanism of cell polarization. 107

Polarized EGFR signaling on the other hand, will lead to reorganization of the cortical 108 actomyosin cytoskeleton by regulating members of the Rho GTPase family, thereby induc- 109 ing signal-dependent cell shape changes and subsequent migration (Chiasson-MacKenize and 110 
McClatchey, 2018; Ridley and Hall, 1992). In order to link signaling activity with morpho- 111 dynamics, we modeled the cell as a viscoelastic cortex surrounding a viscous core (Yang 112 et al., 2008) (Supplementary information), where EGFR signaling dynamics affects cell shape 113 changes through the protrusion/retraction stress and the viscoelastic nature of the cell membrane 114 (Figure 1C).

We first fixed the total EGFR concentration on the cell membrane to a value that corresponds 116 to organization at criticality, and investigated the response of the in silico cell to gradient stim- 117 ulus. In the absence of stimulus, EGFR phosphorylation is uniformly distributed along the cell 118 membrane rendering a symmetrical cell shape (Figure 1D, E). Introducing dynamic gradient 119 stimulus in the simulation (slope changes from steep to shallow over time, Figure 1D, top) led ${ }_{120}$ to rapid polarization of EGFR phosphorylation in the direction of the maximal chemoattrac- $\quad 121$ tant concentration, generating a cell shape with a clear front and back. The polarized signaling 122 state was maintained for a transient period of time after removal of the gradient, corresponding ${ }_{123}$ to manifestation of memory of the localization of the previously encountered signal (Figures $\quad 124$ 1D,E; temporal profile Figure 1F, top). The prolonged polarized state does not result from rem- $\quad 125$ nant ligand-bound receptors $\left(E-E_{p}\right)$ on the plasma membrane, as they exponentially decline ${ }_{126}$ after signal removal (Figure 1F, top). The memory in polarized signaling was also reflected on $\quad{ }_{127}$ the level of the cell morphology, as shown by the difference of normalized cell protrusion area 128 in the front and the back of the cell over time (Figure 1G). Plotting the trajectory that describes ${ }_{129}$ the change of the state of the system over time (state-space trajectory, Figure 1F bottom, movie ${ }_{130}$ $\mathrm{S} 1)$ shows that the temporal memory in EGFR phosphorylation polarization is established due 131 to transient trapping of the signaling state trajectory in state-space. This is typical for the emer- $\quad 132$ gence of metastable "ghost" states (Stanoev et al., 2020; Strogatz, 2018), indicating that the 133 system is maintained away from steady-states. The trapping in the dynamically-metastable ${ }_{134}$ memory state does not hinder sensing of and adapting to subsequent signals. The cell polar- ${ }_{135}$ 
ity is sustained even when the EGF signal is briefly disrupted, and the cell is able to reverse 136 direction of polarization when the signal direction is inverted (Figure 1H, Figures S1C,D). We ${ }_{137}$ next chose in the simulations a higher EGFR concentration on the membrane, such that the ${ }_{138}$ system moves from criticality to organization in the stable inhomogeneous state regime. In this ${ }_{139}$ scenario, even a transient signal induces switching to the polarized state that is permanently 140 maintained, generating a long-term memory of the direction on the initial signal. Thus, the ${ }_{141}$ cell is insensitive to subsequent stimuli from the same direction, whereas consecutive gradients 142 from opposite directions generate conflicting information that cannot be resolved (Figure S1E). ${ }_{143}$ Organization in the homogeneous, symmetric steady states on the other hand renders cells in- 144 sensitive to the extracellular signals (Figure S1F,G). These response features for organization 145 in the stable steady state regimes resemble the finding of previously published models: such ${ }_{146}$ models cannot simultaneously capture memory in polarization along with continuous adapta- ${ }_{147}$ tion to novel signals, or require fine-tuning of kinetic parameters to explain the experimentally 148 observed cell behavior (Levchenko and Iglesias, 2002; Levine et al., 2002; Mori et al., 2008; 149 Goryachev and Pokhilko, 2008; Beta et al., 2008; Xiong et al., 2010; Trong et al., 2014). This 150 demonstrates that organization at criticality, in a vicinity of a $S N_{P B}$, is a unique mechanism for ${ }_{151}$ processing changing signals.

\section{Cells display temporal memory in polarized receptor phos- ${ }_{153}$ phorylation resulting from a dynamical "ghost"

To test experimentally whether cells maintain memory of the direction of previously encoun- ${ }_{155}$ tered signals and what is the duration of this effect, epithelial breast cancer-derived MCF7 156 cells were subjected for $1 \mathrm{~h}$ to a stable gradient of fluorescently tagged EGF-Alexa647 (EGF $\left.{ }^{647}\right){ }_{157}$ with a maximal amplitude of $10 \mathrm{ng} / \mathrm{ml}$ applied from the top of the chamber in a computer- ${ }_{158}$ programmable microfluidic device (Figures 2A,B). EGFR phosphorylation at the plasma mem- 159 
brane was quantified during and for $3 \mathrm{~h}$ after gradient wash-out by determining the rapid translo- $\quad 160$ cation of mCherry-tagged phosphotyrosine-binding domain (PTB ${ }^{m C h e r r y}$ ) to phosphorylated 161 tyrosines 1086/1148 of ectopically expressed EGFR-mCitrine (EGFR ${ }^{\text {mCitrine }}{ }^{\text {}}$ ) using ratiomet- ${ }_{162}$ ric imaging (Offterdinger et al., 2004)(Methods). Due to the low endogenous EGFR levels in 163 MCF7 cells, the expression range of EGFR ${ }^{\text {mCitrine }}$ was set to mimic the endogenous receptor 164 range in the related MCF10A cell line, such that both cell lines have equivalent signaling prop- 165 erties of downstream effector molecules (Stanoev et al., 2018), and were therefore used in a 166 complementary way in this study.

Kymograph analysis of EGFR ${ }^{\text {mCitrine }}$ phosphorylation at the plasma membrane of sin- ${ }_{168}$ gle cells showed polarization in gradient of $\mathrm{EGF}^{647}$ (Figure 2C, Figures. S2A-D), as shal- ${ }_{169}$ low as $10 \%$ between front and back of the cell. Only few cells manifested basal or sym- 170 metric EGFR ${ }^{\text {mCitrine }}$ phosphorylation distribution upon gradient stimulation (Figures S2A- 171 B,E). Quantifying the fraction of plasma membrane area with polarized EGFR ${ }^{\text {mCitrine }}{ }^{\text {phos- }} 172$ phorylation revealed that the polarization persisted $\sim 40 \mathrm{~min}$ on average after gradient re- ${ }_{173}$ moval $([4-159 \mathrm{~min}]$ Figures 2D,E; Figure S2F). In order to identify whether the experi- 174 mentally observed memory results from a dynamically metastable (transiently stable) signal- $\quad 175$ ing state, we next reconstructed the state-space trajectory from the measured single-cell tem- 176 poral EGFR ${ }^{m C i t r i n e}$ posphorylation profile using Takens's delay embedding theorem (Takens, 177 1980)(Methods). Trajectory trapping in a state-space area different than that of the polar- 178 ized and basal steady states characterized the memory phase, corroborating that the memory 179 in EGFR ${ }^{m C i t r i n e}$ phosphorylation polarization emerges from a $S N_{P B}$ "ghost" that maintains 180 the system away from the steady-states (compare Figure $2 \mathrm{~F}$ to $1 \mathrm{~F}$, movie S2). Although the ${ }_{181}$ memory doesn't result from a stable state, it enables to maintain memory of the polarized cell 182 morphology even after gradient removal. This is reflected through the exemplary temporal 183 evolution of the cell protrusion area in direction of the gradient (Figure 2G, memory duration 184 

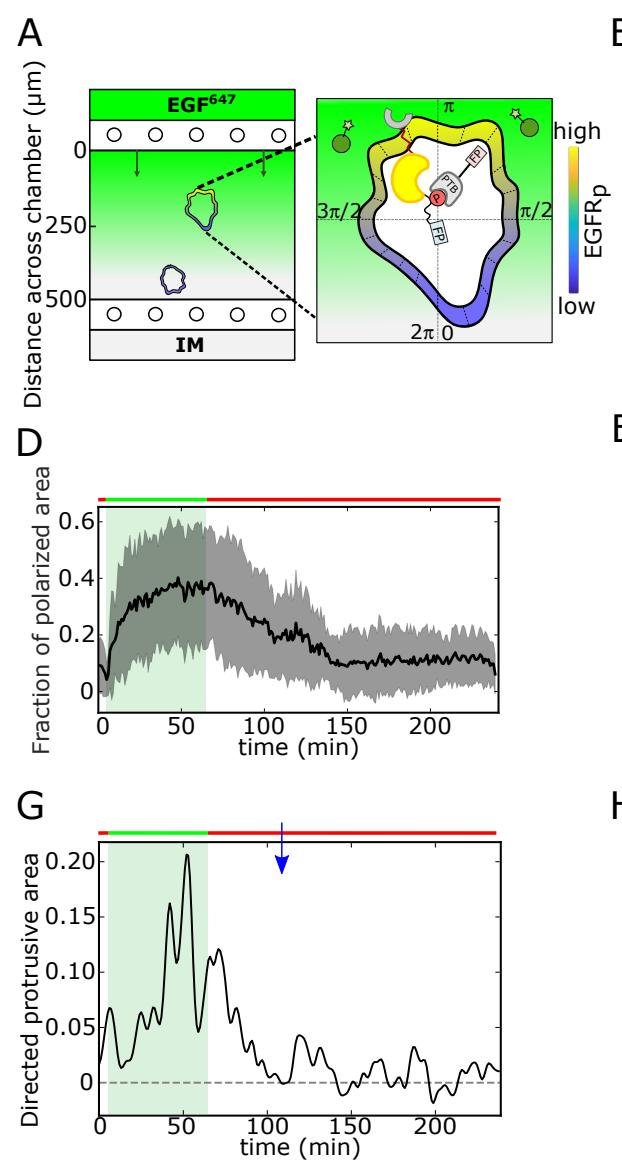

B

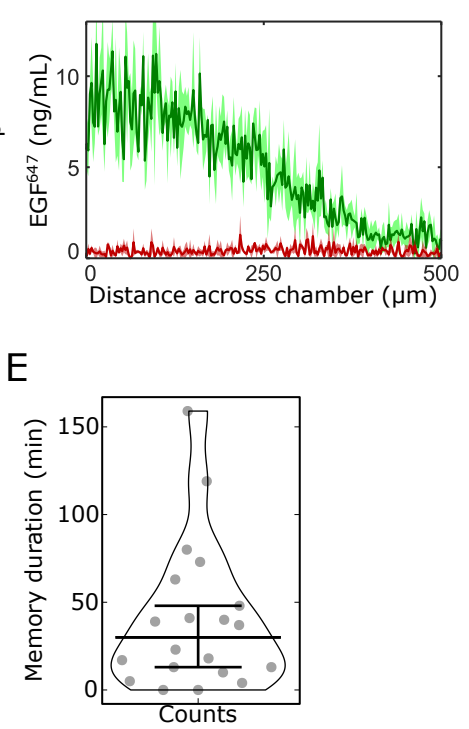

$\mathrm{H}$

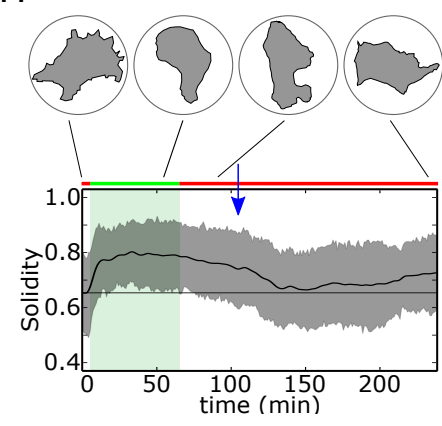

C

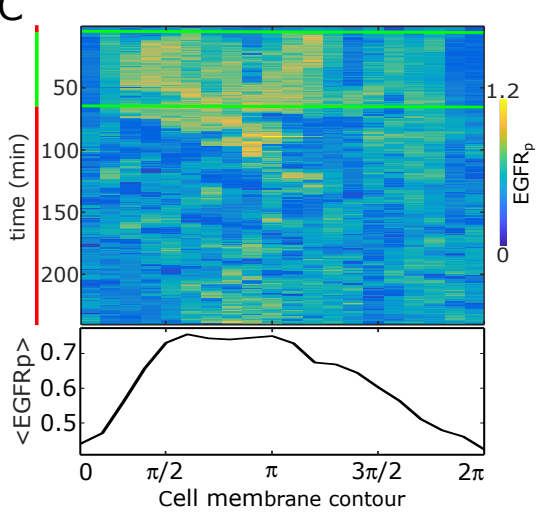

$\mathrm{F}$
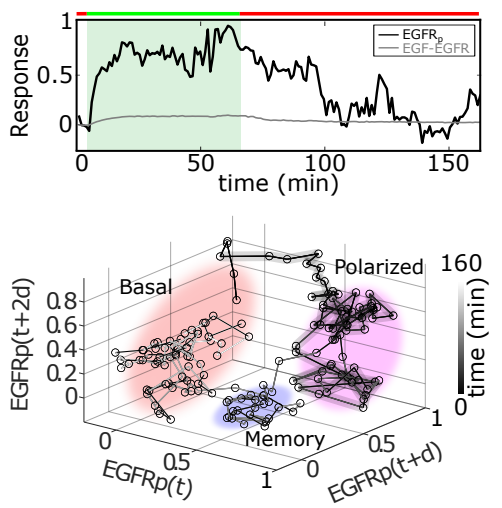

Figure 2. Single-cell molecular memory in polarized EGFR ${ }^{m C i t r i n e}$ phosphorylation from dynamical state-space trapping. A, Scheme of microfluidic $\mathrm{EGF}^{647}$-gradient experiment; Zoom: single-cell measurables. Cell membrane contour $[0,2 \pi]$ (20 segments). PTB - phosphotyrosine binding domain, $F P /$ star symbol - fluorescent protein, $E G F R_{p^{-}}$phosphorylated EGFR $^{m C i t r i n e}$. Remaining symbols as in Figure 1B. B, Quantification of EGF $^{647}$ gradient profile (at $60 \mathrm{~min}$, green) and after gradient wash-out (at $65 \mathrm{~min}$, red). Mean \pm s.d., N=4. C, Exemplary quantification of, Top: single-cell $E G F R_{p}$ kymograph. Data was acquired at $1 \mathrm{~min}$ intervals in live MCF7-EGFR ${ }^{m C i t r i n e}$ cells subjected for $60 \mathrm{~min}$ to an $\mathrm{EGF}^{647}$ gradient. Other examples in Figure S2D. Bottom: respective spatial projection of $E G F R_{p}$. Average using a moving window of 7 bins is shown. Mean \pm s.d. from $n=20, N=7$ in Figure S2C. D, Average fraction of polarized plasma membrane area (mean \pm s.d.). In $\mathbf{D}, \mathbf{E}$ and $\mathbf{H}, \mathrm{n}=20, \mathbf{N}=7$. $\mathbf{E}$, Quantification of memory duration in single cells (median \pm C.I.). F, Top: Temporal profiles of phosphorylated $E G F R^{m C i t r i n e}$ (black) and $E G F^{647}-E G F R^{m C i t r i n e}$ (grey) corresponding to C. Bottom: Corresponding reconstructed state-space trajectory (movie S2) with denoted trapping state-space areas (colored). Thick/thin line: signal presence/absence. $\mathrm{d}$ - embedding time delay. 
G, Exemplary quantification of morphological changes, directed cell protrusion area, for the cell in C. Memory duration: 43min. H, Averaged single-cell morphological changes (Solidity, mean \pm s.d.). Average memory duration: $40 \mathrm{~min}$. Top insets: representative cell masks at distinct time points. In D, F-H, green shaded area: $\mathrm{EGF}^{647}$ gradient duration; green/red lines: stimulus presence/absence. Blue arrow: end of memory. See also Figure S2.

$\sim 43 \mathrm{~min}$ ). On average, single epithelial cells maintained the polarized cell shape $\sim 40 \mathrm{~min} \quad 185$ after signal removal (Figure 2H, Methods). The average duration of memory in the polarized 186 cell morphology therefore directly corresponds to the average memory duration in signaling, 187 suggesting that it will be also reflected as memory in directed cell migration. 188

\section{Transient memory in cell polarization is translated to tran- 189 sient memory in directional migration

To test the phenotypic implications of the transient memory in cell polarization, we analyzed 191 the motility features of the engineered MCF7-EGFR ${ }^{\text {mCitrine }}$, as well as of MCF10A cells at 192 physiological EGF concentrations. Cells were subjected to a 5 h dynamic EGF ${ }^{647}$ gradient that 193 was linearly distributed within the chamber, with $\mathrm{EGF}^{647}$ ranging between $25-0 \mathrm{ng} / \mathrm{ml}$, allowing 194 for optimal cell migration (Figure S3A). The gradient steepness was progressively decreased 195 in a controlled manner, rendering an evolution towards a $\sim 50 \%$ shallower gradient over time 196 (Figure S3B). Automated tracking of single-cell's motility trajectories was performed for $14 \mathrm{~h}$ in $\quad 197$ total. MCF7-EGFR ${ }^{\text {mCitrine }}$, as well as MCF10A cells migrated in a directional manner towards 198 the $\mathrm{EGF}^{647}$ source (Figure 3A- and Figure S3C,D - left, green trajectory parts). This directed ${ }_{199}$ migration persisted for transient period of time after the gradient wash-out (Figure 3A- and 200 Figure S3C,D - left, red trajectory parts, movie S3), indicating that cells maintain memory of ${ }^{201}$ the location of previously encountered source. After the memory phase, the cells transitioned 202 to a migration pattern equivalent to that in the absence of a stimulus (Figure 3A right, Figures ${ }^{203}$ S3C,D middle). Uniform stimulation with $20 \mathrm{ng} / \mathrm{ml} \mathrm{EGF}^{647}$ did not induce directed migration ${ }^{204}$ 
in either of the cell lines, although the overall migration distance was increased (Brueggemann 205 et al., 2021) (Figures S3C,D, right). Quantification of the directionality of single cells' motion, 206 that is defined as the displacement over travelled distance, showed that for MCF10A cells it 207 was significantly higher during the gradient stimulation $(5 \mathrm{~h})$ as compared to no- or uniform- 208 stimulation case (Figure 3B). Moreover, the directionality estimated in the 9h time-frame after 209 the gradient removal was greater than the one in continuous stimulus absence, corroborating 210 that cells transiently maintain memory of the previous direction of migration. 211

This was also reflected in the projection of the cell's relative turning angles $(\cos \theta)$ esti- 212 mated along the gradient direction $(\pi)$ at each time point (Figure S4A), representing the angular ${ }_{213}$ alignment of the cells to the source direction. The cellular migration trajectories aligned with 214 the source direction ( $\cos \theta$ approached 1) during, and maintained this temporally after gradient 215 removal, before returning to a migration pattern characteristic for stimulus absence or during 216 uniform stimulation ( $\cos \theta \approx 0$, Figure 3C top, Figure S4B). Calculating the similarity between 217 the Kernel Density distribution Estimate (KDE) of the angular alignment distributions at each 218 point in the gradient series with that in continuous stimulus absence, showed that the distri- ${ }_{219}$ butions approach each other only $\sim 50 \mathrm{~min}$ after the gradient removal (Figure 3C, bottom). 220 Additionally, the calculated similarity between the KDE distributions during the gradient (5h) 221 and the 50min memory period further corroborated this finding (Figure S4C). The average 222 memory phase in directional motility thus corresponds to the time-frame in which the memory 223 in polarized EGFR ${ }^{\text {mCitrine }}$ phosphorylation and cell shape is maintained (Figures 2C-H), indi- ${ }_{224}$ cating that the metastable signaling state is translated to a stable prolonged migration response 225 after gradient removal.

To investigate whether the motility patterns during the gradient and the memory phase have ${ }_{227}$ equivalent characteristics, we fitted the motility data using a modified Ornstein-Uhlenbeck pro- ${ }_{228}$ cess (Uhlenbeck and Ornstein, 1930; Svensson et al., 2017) and used the extracted migration 229 

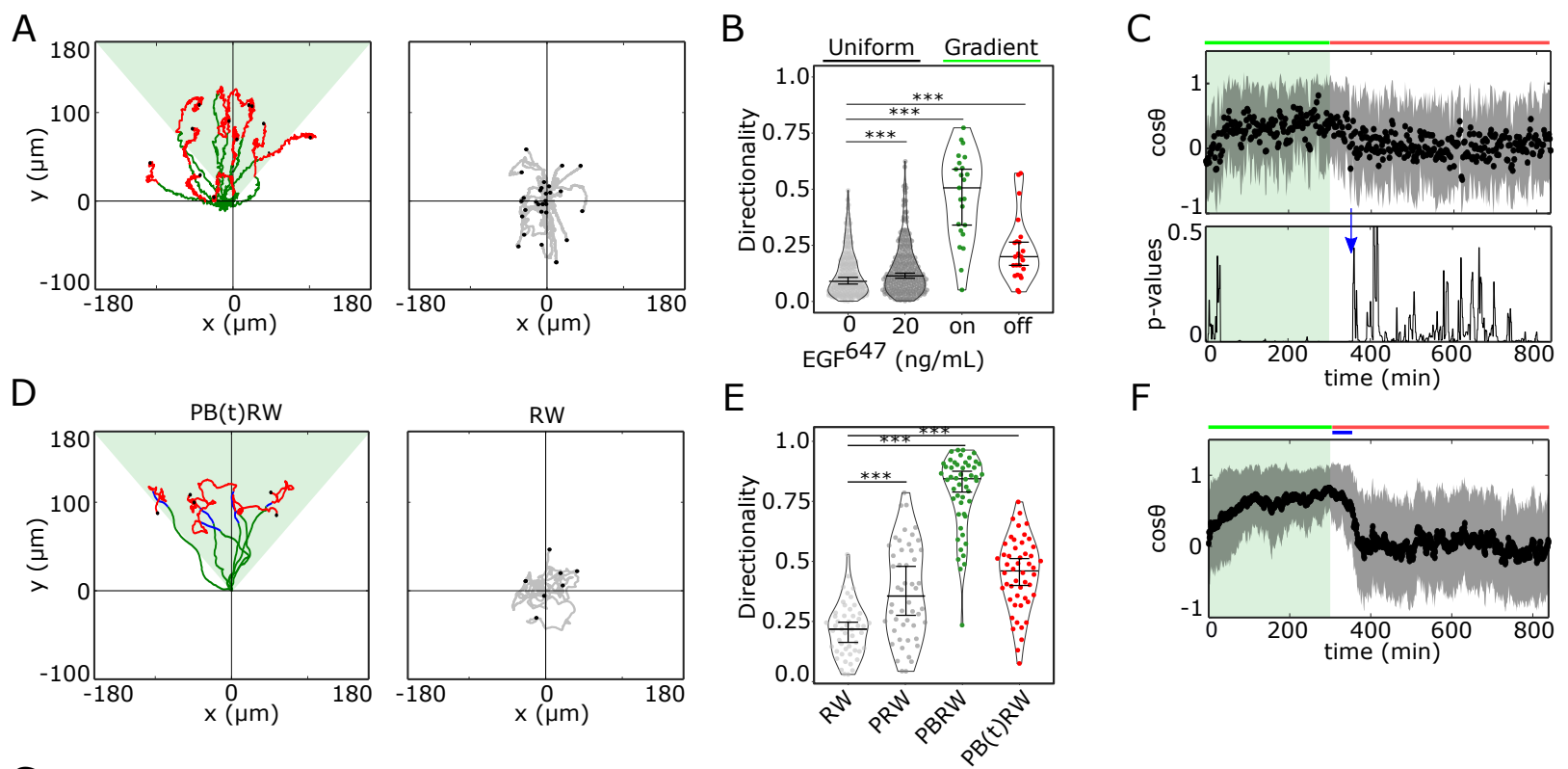

$\mathrm{F}$

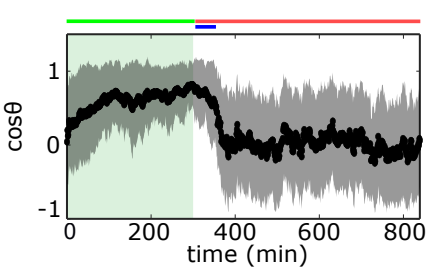

G
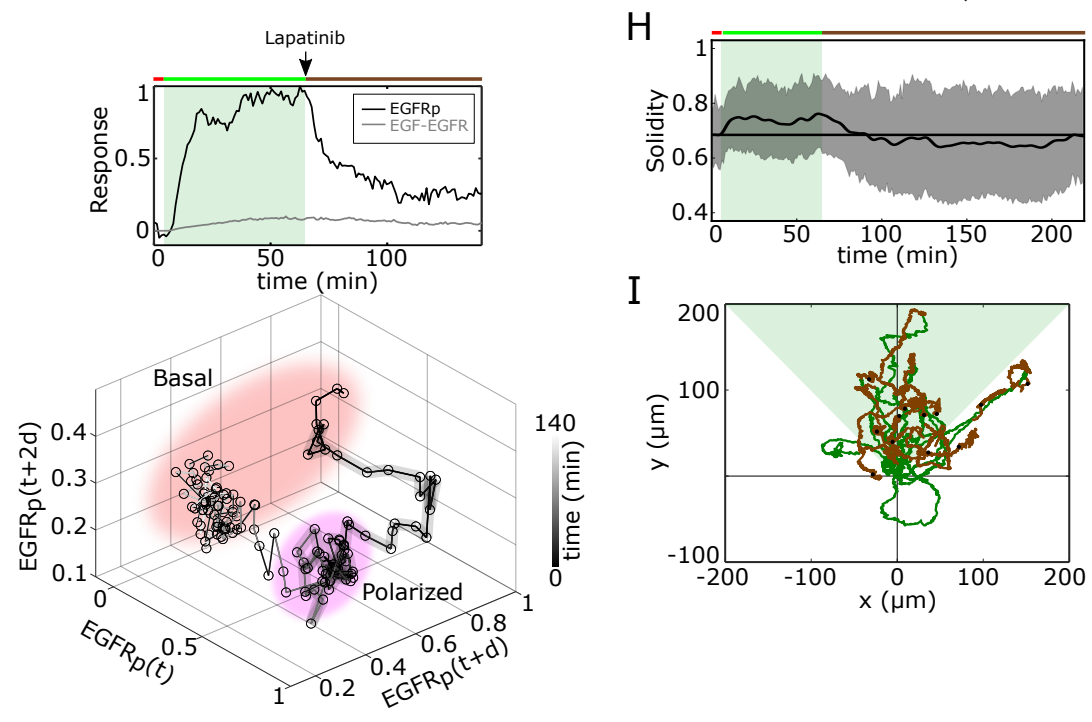

I

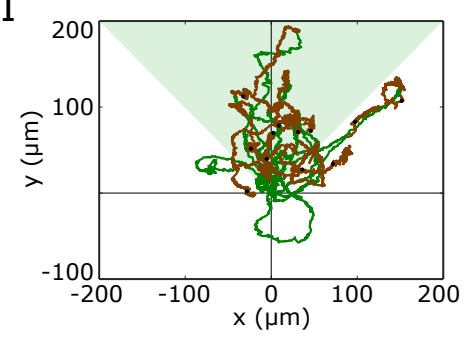

J

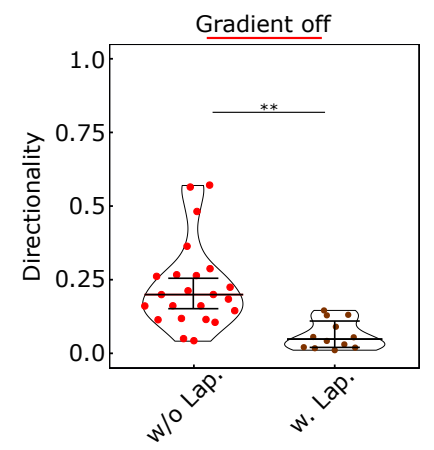

Figure 3. Cells display memory of recently encountered signals. A, Left: representative MCF10A single-cell trajectories. Green - 5h during and red line - $9 \mathrm{~h}$ after dynamic EGF ${ }^{647}$ gradient (shaded). Exemplary cell in movie S3. Right: Same as in A, only 14 h in continuous $\mathrm{EGF}^{647}$ absence. Black dots: end of tracks. 
B, Directionality (displacement/distance) in MCF10A single-cell migration during $14 \mathrm{~h}$ absence (0ng/ml; $\mathrm{n}=249, \mathrm{~N}=3$ ) or uniform 20ng/ml $\mathrm{EGF}^{647}$ stimulation $(\mathrm{n}=299, \mathrm{~N}=3)$; $5 \mathrm{~h}$ dynamic EGF $^{647}$ gradient (green) and 9h during wash-out (red; $\mathrm{n}=23, \mathrm{~N}=5$ ). $\mathrm{p}$-values: $* * * \mathrm{p} \leq 0.001$, twosided Welch's t-test. Error bars: median $\pm 95 \%$ C.I. C, Top: Projection of the cells' relative turning angles (mean \pm sd; $n=23, N=5$ ) during (green shaded) and after 5h dynamic EGF ${ }^{647}$ gradient. Green/red lines: stimulus presence/absence. Bottom: Kolmogorov-Smirnov (KS) test p-values depicting end of memory in directional migration (arrow, $t=350 \mathrm{~min}$ ). KS-test estimated using 5 time points window. For A-C, data sets in Figures S3D, S4A-C. D, Representative in silico single-cell trajectories (Methods). Left: PB(t)RW: Persistent biased random walk, bias is a function of time (green/blue trajectory part - bias on). Right: RW: random walk. E,Corresponding directionality estimates from $\mathrm{n}=50$, data in Figure S4D. PRW: persistent random walk. p-values: $* * * \mathrm{p} \leq 0.001$, two-sided Welch's t-test. Error bars: median $\pm 95 \%$ C.I. F, Same as in C, only from the synthetic $\mathrm{PB}(\mathrm{t}) \mathrm{RW}$ trajectories. G, Top: Exemplary profiles of $E G F R_{p}$ (black) and $E G F-E G F R$ (grey) in live MCF7-EGFR ${ }^{\text {mCitrine }}$ cell subjected to $1 \mathrm{~h} \mathrm{EGF}^{647}$ gradient (green shading), and $4 \mathrm{~h}$ after wash-out with $1 \mu \mathrm{M}$ Lapatinib. Mean \pm s.d. from $\mathrm{n}=9, \mathrm{~N}=2$ in Figure $\mathrm{S} 4 \mathrm{H}$. Bottom: Corresponding reconstructed state-space trajectory with state-space trapping (colored) (Methods. movie S4). H, Average solidity in MCF7-EGFR ${ }^{m \text { Citrine }}$ cells subjected to experimental conditions as in $\mathbf{G}$. Mean \pm s.d. from $n=9, N=2$ cells. I, MCF10A single-cell trajectories quantified $5 \mathrm{~h}$ during (green) and $9 \mathrm{~h}$ after (orange) dynamic $\mathrm{EGF}^{647}$ gradient (shading) wash-out with $3 \mu \mathrm{M}$ Lapatinib. $\mathrm{n}=12, \mathrm{~N}=5$. See also movie S5. J, Directionality in single-cell MCF10A migration after gradient wash-out with (brown, $\mathrm{n}=12, \mathrm{~N}=5$ ) and without Lapatinib (red, $\mathrm{n}=23$, $\mathrm{N}=5$ ). p-values: $* * \mathrm{p} \leq 0.01, \mathrm{KS}$-test. Error bars: median $\pm 95 \%$ C.I. See also Figures S3 and S4.

parameters to generate synthetic single-cell trajectories (Methods). In absence of stimulus, the cellular motion resembled a random walk process (RW: Figure 3D right, Figures S4D,E mid- ${ }_{231}$ dle), persistent random walk (PRW) was characteristic for the uniform stimulation case (Figure ${ }_{232}$ S4D, E right), whereas biased PRW described the migration in gradient presence (PBRW, Fig- ${ }_{233}$ ure 3D- and Figure S4D, left, green trajectory part). Extending the bias duration during the ${ }_{234}$ interval of the experimentally observed memory phase $(\mathrm{PB}(\mathrm{t}) \mathrm{RW})$ was necessary to reproduce 235 the transient persistent motion after gradient removal (Figure 3D- and Figure S4D, left, blue ${ }_{236}$ trajectory part; Figures 3E,F; Figure S4F). Altogether, these results demonstrate that epithelial ${ }_{237}$ cells transiently maintain a memory of previous signal location and thereby display directed ${ }_{238}$ motility equivalent to that in the presence of a gradient, before reverting to a random walk 239 migration pattern. 
To corroborate the link between memory in polarized receptor activity and memory in di- 241 rectional migration, we quantified EGFR ${ }^{\text {mCitrine }}$ phosphorylation polarization in the MCF7- 242 $\mathrm{EGFR}^{\text {mCitrine }}$, as well as directional migration of MCF10A cells, when cells were subjected ${ }_{243}$ to an ATP analog EGFR inhibitor Lapatinib (Bjorkelund et al., 2012) during gradient wash- 244 out. The exemplary single-cell kymograph and EGFR ${ }^{\text {mCitrine }}$ phosphorylation temporal profile 245 demonstrate that the phosphorylation response exponentially decays upon Lapatinib addition, 246 resulting in a clear absence of transient memory in EGFR ${ }^{m \text { Citrine }}$ phosphorylation polarization ${ }_{247}$ (Figure 3G top, Figures S4G,H). This is further reflected in the reconstructed state-space tra- ${ }_{248}$ jectory that smoothly transits from the polarized to the basal activity state, without the transient 249 state-space trapping that was characteristic for the memory state emerging from the dynamical 250 "ghost" (compare Figure 3G bottom to 2F, movie S4). The absence of memory in EGFR ${ }^{\text {mCitrine }} 251$ phosphorylation was also reflected in absence of transient memory in morphological changes 252 after stimulus removal (Figure 3H). In the MCF10A migration assay, cells directly switched 253 to RW migration pattern upon gradient wash-out with Lapatinib, as shown through the direc- 254 tionality quantification after gradient removal (Figures 3I,J; movie S5). Equivalent single-cell 255 motility trajectories could be mimicked with the PB(t)RW simulation, where the bias dura- 256 tion corresponded to the duration of the gradient (Figures S4I,E). This shows that the transient 257 memory arising from a metastable "ghost" signaling state is a core dynamical feature under- 258 lying transient memory in directional motility, and cannot be explained with slow relaxation 259 kinetics in receptor dephosphorylation. 260

\section{Molecular working memory enables cells to navigate in $\mathbf{d y}-{ }_{261}$ namic chemoattractant fields

To test whether the identified memory enables cellular navigation in environments where sig- 263 nals are disrupted but also change over time and space, we subjected cells in the simulations 264 
A

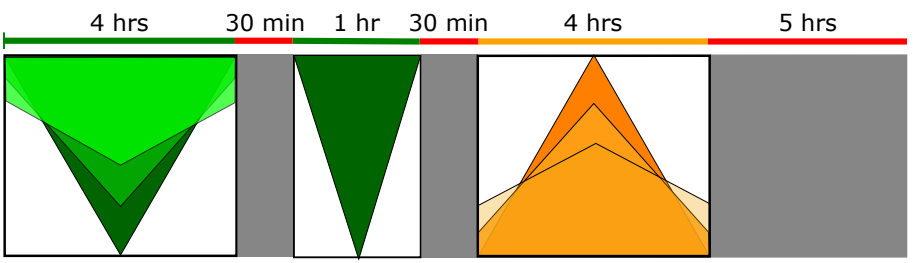

B

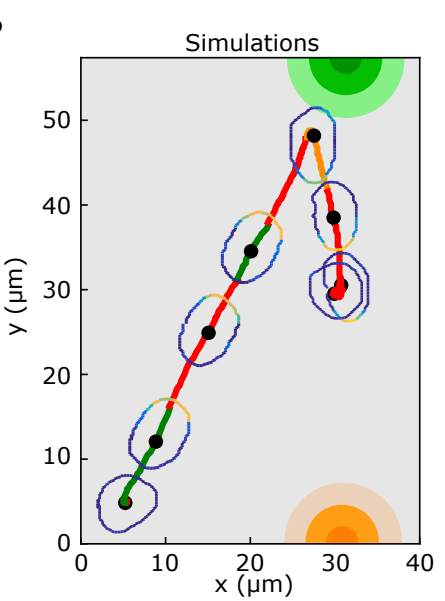

C

\section{$\mathrm{D}$}

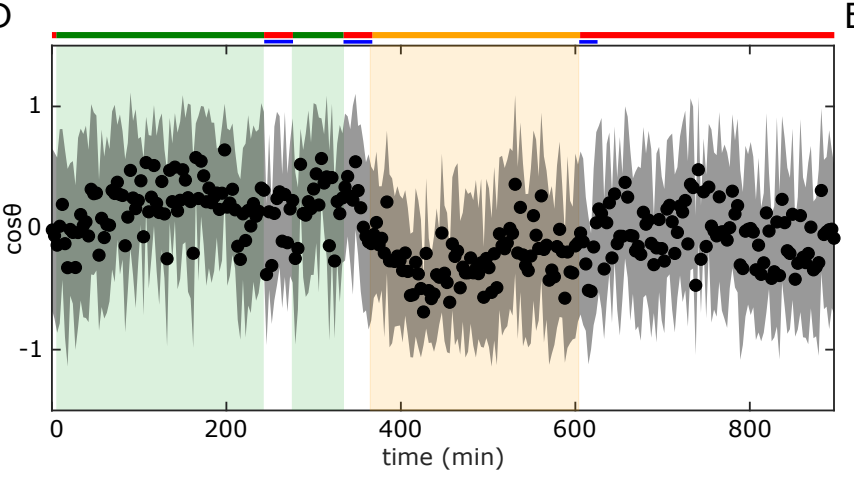

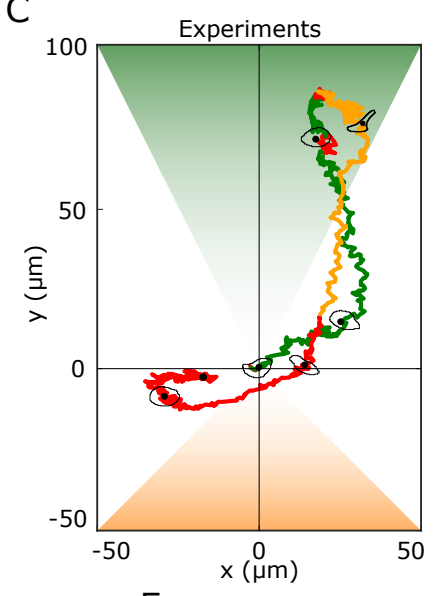

$\mathrm{E}$

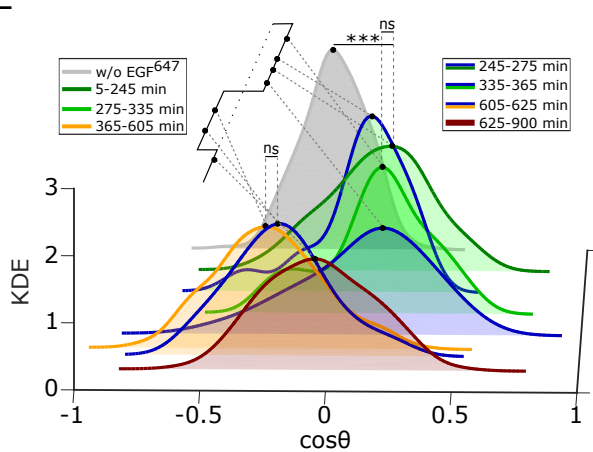

Figure 4. History-dependent single-cell migration in changing chemoattractant field. A, Scheme of dynamic spatial-temporal growth factor field implemented in the simulations and experiments. Green(orange)/red: gradient presence/absence. B, In silico cellular response to the sequence of gradients as depicted in $\mathbf{A}$, showing changes in EGFR activity, cellular morphology and respective motility trajectory over time. Trajectory color coding corresponding to that in (A), cell contour color coding with respective $E_{p}$ values as in Figure 1E. Cell size is magnified for better visibility. See also movie S6. C, Representative MCF10A single-cell trajectory and cellular morphologies at distinct time-points, when subjected to dynamic $\mathrm{EGF}^{647}$ gradient field as in A. Trajectory color coding corresponding to that in A. See also movie S8. D, Projection of cells' relative turning angles $(\cos \theta)$ depicting their orientation towards the respective localized signals. Mean \pm s.d. from $n=12, N=5$ is shown. Data in Figure S5E. E, Corresponding kernel density estimates (intervals and color coding in legend). p-values: $* * *, \mathrm{p} \leq 0.001$, ns: not significant, KS-test. See also Figure S5. 
and experiments to a changing growth factor field. The field was generated by a sequence of 265 signals, starting with a dynamic gradient whose steepness changed over time, that was tem- 266 porary disrupted for a time interval shorter than the interval of memory in cell polarization, 267 followed by a second static gradient in the same direction, that after an equivalent disruption 268 period was followed by a third dynamic gradient in the opposite direction (Figure 4A). The ${ }_{269}$ in silico migration simulations showed that the cell can sense the initial dynamic gradient and 270 polarizes in the direction of maximal attractant concentration, resulting in directed migration 271 (Figure 4B, Figure S5A, movie S6). The simulations also predicted that the memory of the pre- 272 viously encountered signal localization enables maintaining robust directional migration even 273 when the signal was disrupted, while still remaining sensitive to the newly emerging signal 274 from the opposite direction. In the simulation, the in silico cell rapidly adapted the orientation 275 when encountering the third signal with opposite localization, demonstrating that the proposed 276 mechanism can also account for prioritizing newly encountered signals. Such a dynamic mem- 277 ory which enables information of previous signals to be temporally maintained while retaining 278 responsiveness to upcoming signals and thereby manipulate the stored information, in neuronal 279 networks is described as a working memory (Atkinson and Shiffrin, 1968). On the other hand, 280 the simulations also showed that the long-term memory resulting from organization in the stable 281 inhomogeneous steady state regime, hindered cellular adaptation to a changing gradient field. 282 The initial dynamic gradient shifted the system to the stable polarization steady state where it 283 was maintained in a long-term, such that sensitivity to upcoming signals from the same direc- ${ }_{284}$ tion was hindered. Even more, in the simulations, the cell could not resolve the conflicting 285 information from a subsequent gradient from the opposite direction as the signals induced high 286 receptor activity on the opposed cell sides, resulting in ending the migration (Figures S5B,C, 287 movie S7).

We next tested these predictions experimentally, by establishing an equivalent dynamic 289 
EGF $^{647}$ spatial-temporal field in a controlled manner in the microfluidic chamber, and quanti- 290 fied the migratory profile of MCF10A cells (Figure S5D). The MCF10A cells sensed the initial 291 dynamic gradient field and migrated in the direction of the largest chemoattractant concentra- 292 tion, maintaining the directionality even when the signal was temporary disrupted. Despite the ${ }_{293}$ memory in cell polarization, cells remained responsive and adapted the duration of directional 294 migration when presented with a second static gradient from the same direction, and subse- 295 quently prioritized the third, newly encountered signal with opposed orientation (exemplary 296 trajectory in Figure 4C, movie S8, Figure S5E). The temporal memory in directional migration 297 as well as the continuous adaptation of MCF10A cells to novel cues was also reflected in the 298 projection of the cell's relative turning angles (Figure 4D), whereas the respective KDE dis- ${ }^{299}$ tributions derived from the subsequent time-intervals of gradient presence/absence corroborate 300 that cells maintain the same migration characteristics within the memory intervals as during the 301 gradient phase (Figure 4E). These results demonstrate that cells navigate in changing gradient 302 fields by utilizing a molecular mechanism of working memory that is an intrinsic feature of 303 receptor tyrosine kinase networks. 304

\section{Discussion}

Our data establishes that mammalian cells use a mechanism of working memory to navigate in 306 complex environments where the chemical signals are disrupted or vary over time and space. 307 Even though persistent migration of eukaryotic cells in absence of signals was previously ob- ${ }_{308}$ served (Skoge et al., 2014; Albrecht and Petty, 1998; Prentice-Mott et al., 2016), its underlying 309 mechanism and the implications for navigation in changing environments have not been elu- 310 cidated. The mechanism of transient memory we report here is realized on a molecular level, 311 by storing information about direction of previously encountered signals through maintaining 312 a prolonged polarized phosphorylation state of receptor tyrosine kinases. Dynamically, the ${ }_{313}$ 
prolonged polarized state emerges for organization of receptor networks at criticality, where a 314 slow-escaping remnant from the attractor state or a dynamical "ghost" is generated. The "ghost" 315 maintains the system away from steady state, suggesting that in migrating cells, the information 316 about previously encountered signals can be encoded in the transient state-space trajectories 317 rather than the steady-states of the protein interaction network. Our simulations and migration 318 experiments show that this encoding via transient states is necessary to ensure the ability of cells 319 to adapt to changes in the external environment, while maintaining memory of previous signals. $\quad 320$ Our work furthermore suggest that this general mechanism of a system poised at criticality can 321 explain a wide range of biologically relevant scenarios, from the integration of temporally and 322 spatially varying signals, to how extracellular information is transformed into guidance cues for 323 memory-directed migration. Memory-guided navigation is advantageous when migration must 324 be realized over long and complex trajectories through dense tissues where the chemical cues 325 are disrupted or only locally organized. 326

For neuronal networks, short-term memory is a main requirement to integrate temporal 327 dependencies from changing signals (Hochreiter and Schmidhuber, 1997; Maass et al., 2000). 328 We have demonstrated here that the transient memory in cell polarization and therefore the ${ }_{329}$ capabilities of cells to navigate in a complex environment are an emergent feature of receptor 330 networks organized at criticality, and cannot be explained using computations with stable states. $\quad 331$ It would be of interest to study whether receptor networks are self-organized at criticality, or 332 these features arose through evolution as a means for optimizing the computational capabilities 333 of cells. The identification of a molecular working memory also opens avenues of research 334 in the single-cell migration and tissue homeostasis to study whether cells can integrate and 335 interpret even sub-threshold environmental signals, leading to release of cells from a tissue and 336 long-distance single cell migration, as during cancer metastasis. 337 


\section{Acknowledgments}

The authors thank Angel Stanoev for initial analysis of the EGFR polarity model, critical dis- 339 cussion during the project, as well as valuable comments on the manuscript, Manish Yadav and 340 Monika Scholz for critical reading of the manuscript and valuable suggestions, and Frédéric ${ }_{341}$ Paquin-Lefebvre for valuable suggestions on the realization of the reaction-diffusion simula- 342 tions. All of the experiments were carried in the lab of Philippe Bastiaens, and we are particu- 343 larly grateful for the opportunity to be part of that engaging and critical community where we 344 could learn and develop this project. We especially thank P. Bastiaens for numerous critical 345 discussion and suggestionsthat were crucial throughout the project, as well as for detailed com- ${ }_{346}$ ments that significantly helped us to improve the manuscript. Data and code availability: The ${ }^{347}$ data can be obtained from the corresponding author upon reasonable request. The codes will be ${ }_{348}$ made publicly available upon acceptance. Funding: The project was funded by the Max Planck 349 Society, partially through the Lise Meitner Excellence Program. 350

\section{Author contributions}

A.K. conceptualized and supervised the project; A.P.N. and A.K. developed the theoretical 352 description; A.P.N. performed the analytical and numerical analysis; A.D. performed most of ${ }_{353}$ the experiments with help of A.P.N.; A.D., A.P.N. and R.L. analyzed the data. A.K. wrote the ${ }^{354}$ manuscript with help of A.D., A.P.N. and R.L.

\section{Competing Interests statement}

The authors declare no competing interests. 


\section{Materials and Methods}

\subsection{Cell Culture}

MCF7 cells (sex: female, ECACC, Cat. No. 86012803) were grown at $37^{\circ} \mathrm{C}$ and $5 \% \mathrm{CO}_{2} \quad 360$ in Dulbecco's Eagle's medium (DMEM) (PAN-Biotech, Germany), supplemented with 10\% 361

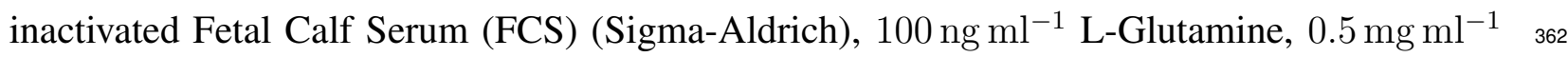
non-essential amino acids, $100 \mu \mathrm{g} \mathrm{ml} \mathrm{m}^{-1}$ penicillin and $100 \mu \mathrm{g} \mathrm{ml}^{-1}$ streptomycin (PAN-Biotech, 363 Germany). Serum starvation was performed by culturing the cells in DMEM supplemented with 364 $0.5 \% \mathrm{FCS}, 100 \mu \mathrm{g} \mathrm{ml}^{-1}$ penicillin and $100 \mu \mathrm{g} \mathrm{ml} \mathrm{m}^{-1}$ streptomycin (PAN-Biotech, Germany). 365 MCF10A cells (sex: female, ATCC-CRL 10317) were grown at $37^{\circ} \mathrm{C}$ and $5 \% \mathrm{CO}_{2}$ in Mammary 366 Epithelial Cell Growth Basal medium (MEBM from Lonza Pharma \& Biotech), supplemented ${ }_{367}$ with 5\% Horse Serum (HS) (Invitrogen), $20 \mathrm{ng} \mathrm{mL}{ }^{-1}$ EGF (Sigma-Aldrich), $0.5 \mathrm{mg} \mathrm{mL}^{-1}$ hy- 368 drocortisone (Sigma-Aldrich), $100 \mathrm{ng} \mathrm{ml}^{-1}$ cholera toxin (Sigma-Aldrich), $10 \mu \mathrm{g} \mathrm{mL}^{-1}$ insulin 369 (Sigma-Aldrich), $100 \mu \mathrm{g} \mathrm{mL} \mathrm{mL}^{-1}$ penicillin and $100 \mu \mathrm{g} \mathrm{mL} \mathrm{m}^{-1}$ streptomycin. Serum starvation was 370 performed by culturing the cells in the DMEM supplemented with $0.5 \% \mathrm{HS}, 0.5 \mathrm{mg} \mathrm{mL}^{-1}$ hy- 371 drocortisone (Sigma-Aldrich), $100 \mathrm{ng} \mathrm{ml}^{-1}$, cholera toxin (Sigma-Aldrich) $100 \mu \mathrm{g} \mathrm{mL}^{-1}$ peni- ${ }_{372}$ cillin and $100 \mu \mathrm{g} \mathrm{mL}^{-1}$ streptomycin. MCF7 and MCF10A cells were authenticated by Short 373 Tandem Repeat (STR) analysis and did not contain DNA sequences from mouse, rat and ham- ${ }^{374}$ ster (Leibniz-Institut DSMZ). Cells were regularly tested for mycoplasma contamination using 375 MycoAlert Mycoplasma detection kit (Lonza).

\subsection{Transfection and cell seeding}

For EGFR ${ }^{\text {mCitrine }}$ polarization experiments, $2.5 \times 10^{5}$ MCF7 cells were seeded per well in a 6- ${ }_{378}$ well Lab-Tek chamber (Nunc) until 80\% confluence was reached. After 9-10 h of seeding, tran- ${ }_{379}$ sient transfection was performed with a total of $1 \mu \mathrm{g}$ of plasmids $\left(E G F R^{m C i t r i n e}, P T B^{m C h e r r y} \quad{ }_{380}\right.$ and $c C b l^{B F P}$ at ratio 4:3:4 by mass) using FUGENE6 (Roche Diagnostics) transfection reagent 381 
and Opti-MEM (Gibco - Thermo Fisher Scientific) according to manufacturer's procedure. All 382 plasmids were generously provided by Prof. P. Bastiaens, MPI of Molecular Physiology, Dort- 383 mund. Cells were incubated for 7-8 h to allow the expression of the transfected proteins prior 384 to experiments. To detach the cells, the growth media was discarded and cells were washed 385 once with DPBS (PAN Biotech) before adding $100 \mu \mathrm{L}$ Accutase (Sigma-Aldrich). After 10 min 386 incubation period at $37^{\circ} \mathrm{C}$ and $5 \% \mathrm{CO} 2$, fresh growth media was added, and the cell density and ${ }_{387}$ viability was measured using cell counter (Vi-CELL XR Cell Viability Analyzer System). After 388 spinning down, the cells were diluted to $10 \times 10^{6}$ cells/ml. The M04-G02 microfluidic gradient 389 plates (Merck Chemicals) were primed for usage by flowing cell culture growth media through 390 the cell chamber for $5 \mathrm{~min}$ and cells were subsequently seeded according to manufacturer's 391 instructions.

For migration experiments with uniform $E G F^{647}$ stimulation, 6-well Lab-Tek plates were ${ }_{393}$ coated with Collagen (Sigma-Aldrich) in 0.1 M Acetic acid (Sigma-Aldrich) for MCF7 394 $\left(100 \mu \mathrm{g} \mathrm{cm}^{-2}\right)$, and Fibronectin (Sigma-Aldrich) in Phosphate-Buffered Saline (DPBS) (PAN- ${ }_{395}$ Biotech) for MCF10A cells $\left(2 \mu \mathrm{g} \mathrm{mL}{ }^{-1}\right)$, and stored in incubator at $37^{\circ} \mathrm{C}$ overnight for evapo- 396 ration. Excessive media was removed and the wells were washed with DPBS before seeding 397 cells. MCF7 cells were seeded and transfected as described above. In the case of MCF10A 398 cells, $1 \times 10^{5}$ cells per well were used for seeding. For migration experiments with gradient 399 EGF $^{647}$ stimulation, MCF7 cells were transferred to the coated M04-G02 microfluidic gradient 400 plates as described above. Before seeding, MCF10A cells were detached from 6 well Lab-Teks ${ }_{401}$ by discarding the growth media and washing once with DPBS (PAN Biotech) before adding ${ }_{402}$ $100 \mu \mathrm{L}$ Accutase (Sigma-Aldrich). After $20-30 \mathrm{~min}$ incubation period at $37^{\circ} \mathrm{C}$ and $5 \% \mathrm{CO} 2,403$ fresh cell growth media was added, and the cell density and viability were measured using a 404 cell counter (Vi-CELL XR Cell Viability Analyzer System). After spinning down, the cells 405 were diluted to $2 \times 10^{6}$ cells $/ \mathrm{ml}$, and subsequently seeded in the microfluidic plates according ${ }_{406}$ 


\subsection{Reagents}

For gradient quantification, Fluorescein (Sigma Aldrich) was dissolved in Dulbecco's modified 409 Eagle's medium (with 25 mM HEPES, without Phenol Red) (PAN Biotech). Imaging media: 410 DMEM without Phenol Red was mixed with 25 mM HEPES. For nuclear staining, $20 \mathrm{mM} 411$ Hoechst 33342 (Thermo Fisher Scientific) was mixed with DPBS and diluted to $2 \mu \mathrm{M}$ working ${ }_{412}$ concentration. EGFR inhibitor Lapatinib (Cayman Chemical, Ann Arbor, MI) was solubilized ${ }_{413}$ in DMSO (Thermo Fisher Scientific) to a stock concentration of $5 \mathrm{mM}$ and stored at $-20^{\circ} \mathrm{C}$. $\quad{ }^{414}$

\subsection{Confocal and wide-field microscopy}

Confocal images were recorded using a Leica TCS SP8i confocal microscope (Leica Microsys- ${ }_{416}$ tems) with an environment-controlled chamber (Life Imaging Services) maintained at $37^{\circ} \mathrm{C} \quad 417$ and HC PL APO 63x/1.2 N.A / motCORR CS2 water objective (Leica Microsystems) or a 418 HC PL FLUOTAR 10x/0.3 N.A. dry objective (Leica Microsystems). mCitrine, mCherry and 419 Alexa647 were excited with a $470 \mathrm{~nm}-670 \mathrm{~nm}$ pulsed white light laser (Kit WLL2, NKT Pho- ${ }_{420}$ tonics) at $514 \mathrm{~nm}, 561 \mathrm{~nm}$ and $633 \mathrm{~nm}$, respectively. BFP and Hoechst 33342 (Thermo Fisher ${ }_{421}$ Scientific) were excited with a $405 \mathrm{~nm}$ diode laser. The detection of fluorescence emission 422 was restricted with an Acousto-Optical Beam Splitter (AOBS): BFP (425 nm-448 nm), Hoechst ${ }_{423}$ $33342(425 \mathrm{~nm}-500 \mathrm{~nm})$, mCitrine (525 nm-551 nm), mCherry (580 nm-620 nm) and Alexa647 ${ }_{424}$ (655 nm-720 nm). Transmission images were recorded at a 150-200\% gain. To suppress laser 425 reflection, Notch filter 488/561/633 was used whenever applicable. When using the dry ob- 426 jective for migration experiments, the pinhole was set to 3.14 airy units and 12-bit images of ${ }_{427}$ $512 \times 512$ pixels were acquired in frame sequential mode with $1 \mathrm{x}$ frame averaging. When using ${ }_{428}$ the water objective for polarization experiments, the pinhole was fixed (1.7 airy units) for all ${ }_{429}$ 
Wide field images were acquired using an Olympus IX81 inverted microscope (Olympus 431 Life Science) equipped with a MT20 illumination system and a temperature controlled $\mathrm{CO}_{2}$ in- ${ }_{432}$ cubation chamber at $37^{\circ} \mathrm{C}$ and $5 \% \mathrm{CO}_{2}$. Fluorescence and transmission images were collected ${ }_{433}$ via a 10x/0.16 NA air objective and an Orca CCD camera (Hamamatsu Photonics). Hoechst ${ }^{434}$ 33342 fluorescence emission was detected between $420 \mathrm{~nm}-460 \mathrm{~nm}$ via DAPI filter, mCitrine ${ }_{435}$ fluorescence emission between 495 nm-540 nm via YFP filter and Alexa647 fluorescence emis- ${ }_{436}$ sion between $705 \mathrm{~nm}-745 \mathrm{~nm}$ via Cy5 filter. The xCellence (Olympus) software was used. ${ }_{437}$

\subsection{Gradient establishment for polarization and migration experiments}

The CellAsic Onix Microfluidic Platform (EMD Millipore) was used for gradient cell migration and EGFR ${ }^{m C i t r i n e}$ phosphorylation polarization experiments. For EGFR ${ }^{m C i t r i n e}$ phosphoryla439 tion polarization experiments, $1 \mathrm{~h}$ gradient stimulation was established using CellASIC ONIX2 ${ }^{441}$ software as follows. (i) Pre-stimulus: Imaging media was flowed from well groups 3 and 4 (Cel- ${ }_{442}$ 1Asic Onix Manual - www.merckmillipore.com/) at low pressure $(2.5 \mathrm{kPa})$ for $5 \mathrm{~min}$. (ii) Gra- ${ }_{443}$ dient establishment: After closing well group 3, pre-loaded EGF ${ }^{647}\left(10 \mathrm{ng} \mathrm{mL}^{-1}\right)$ was flowed ${ }_{444}$ through well group 2 and imaging media from well group 4 at high pressure ( $15 \mathrm{kPa})$ for $15 \min { }_{445}$ (iii) Gradient maintenance: The pressure was reduced to $10 \mathrm{kPa}$ for $45 \mathrm{~min}$. (iv) Washout: Af- ${ }_{446}$ ter closing well groups 2 and 4, imaging media was flowed from well groups 3 and 5 at high ${ }_{447}$ pressure $(15 \mathrm{kPa})$ for $15 \mathrm{~min}$ and maintained at low pressure $(7 \mathrm{kPa})$ for $165 \mathrm{~min}$. For single ${ }_{448}$ gradient migration experiments, this protocol was modified as follows: in step (iii), gradient ${ }_{449}$ maintenance was done for $285 \mathrm{~min}$. In step (iv), maintenance was at low pressure for $585 \mathrm{~min} . \quad 450$ $30 \mathrm{ng} \mathrm{mL}^{-1} \mathrm{EGF}^{647}$ was used. For polarization experiments with inhibitor, the same protocol 451 as for polarization experiments was used, except well group 3 and 5 were filled with $1 \mu \mathrm{M} \mathrm{La-}{ }_{452}$ patinib solution and in step (i) well group 3 was kept closed. For single cell gradient migration ${ }_{453}$ 
experiment with inhibitor, $3 \mu \mathrm{M}$ Lapatinib was used.

For migration experiments under subsequent gradient stimuli / gradient quantification, the ${ }_{455}$ following changes in the steps were used : (ii) well group 2 with $30 \mathrm{ng} \mathrm{mL}{ }^{-1} \mathrm{EGF}^{647} / 2.5 \mu \mathrm{M}{ }_{456}$ Fluorescein was used. (iii) The gradient maintenance was done for $225 \mathrm{~min}$. (iv) Washout: 457 imaging media was flowed from well groups 3 and 4 at high pressure $(15 \mathrm{kPa})$ for $15 \mathrm{~min}$ and 458 maintained at low pressure $(7 \mathrm{kPa})$ for $15 \mathrm{~min}$. (v) Second gradient establishment: After closing ${ }_{459}$ well group 3, $\mathrm{EGF}^{647}\left(30 \mathrm{ng} \mathrm{mL}^{-1}\right) / 2.5 \mu \mathrm{M}$ Fluorescein was flowed from well group 2 and 460 imaging media from well group 4 at high pressure $\left(15 \mathrm{kPa}\right.$ ) for $15 \mathrm{~min}$. (vi) The second gradient ${ }_{461}$ thus formed was maintained by reducing the pressure to $10 \mathrm{kPa}$ for $45 \mathrm{~min}$. (vii) Washout: ${ }_{462}$ imaging media was flowed from well groups 3 and 4 at high pressure $(15 \mathrm{kPa})$ for $15 \min 463$ and maintained at low pressure $(7 \mathrm{kPa}$ ) for $15 \mathrm{~min}$. (viii) Third gradient establishment: After 464 closing well group 4, $\mathrm{EGF}^{647}\left(30 \mathrm{ng} \mathrm{mL}^{-1}\right) / 2.5 \mu \mathrm{M}$ Fluorescein was flowed from well group 465 5 and imaging media from well group 3 at high pressure $(15 \mathrm{kPa})$ for $15 \mathrm{~min}$. (ix) The third 466 reversed gradient was maintained by reducing the pressure to $10 \mathrm{kPa}$ for $225 \mathrm{~min}$. (x) Washout: ${ }_{467}$ imaging media was flowed from well groups 3 and 4 at high pressure $(15 \mathrm{kPa})$ for 15 min and 468 maintained at low pressure $(7 \mathrm{kPa})$ for $285 \mathrm{~min}$.

\subsection{Imaging EGF $R^{\text {mCitrine }}$ phosphorylation polarization and single cell ${ }_{470}$ migration

Transfected MCF7-EGFR ${ }^{\text {mCitrine }}$ cells transferred to M04G-02 gradient plates as described 472 above were incubated for at least $3 \mathrm{~h}$, followed by serum starvation for at least $6 \mathrm{~h}$ before imag- 473 ing. Existing cell media was substituted right before imaging with imaging media. Confocal 474 imaging for multiple positions at 1 min time interval using adaptive auto-focus system and the 475 water objective was performed concurrently during the duration of the experiment using the 476 Leica TCS SP8i. 
For migration experiments under uniform $\mathrm{EGF}^{647}$ stimulation, confocal laser scanning mi- 478

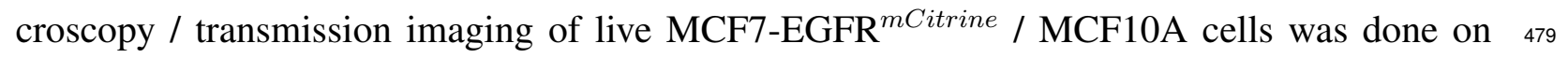
a Leica TCS SP8i or Olympus IX81 for multiple positions at $3 \mathrm{~min}$ and $2 \mathrm{~min}$ time interval 480 respectively, using the 10x dry objective for 14 hours.

\subsection{EGF $^{647} /$ Fluorescein gradient quantification}

hEGF $^{647}$ was generated in the lab of Prof. P. Bastiaens, MPI of molecular Physiology, Dort- ${ }^{483}$ mund, using the His-CBD-Intein-(Cys)-hEGF-(Cys) plasmid (Sonntag et al., 2014), kindly pro- 484 vided by Prof. Luc Brunsveld, University of Technology, Eindhoven. Human EGF was purified 485 from E. coli BL21 (DE3), N-terminally labeled with Alexa647-maleimide as described previ- 486 ously (Sonntag et al., 2014) and stored in PBS at $-20^{\circ} \mathrm{C}$. To quantify the spatial extent of the ${ }_{487}$ $\mathrm{EGF}^{647}$ / Fluorescein gradient, gradients were generated following the protocol described in 488 sub-section 5.5 in plates without cells or matrix coating. Confocal images of Alexa647 / GFP 489 channel were acquired at 1 min interval. A rectangular region of interest (including the perfu- 490 sion channels and the culture chamber) was used to obtain an averaged pixel intensity profile 491 using FIJI at each time point. This spatial profile was averaged across multiple experiments and 492 then scaled with the mean intensity value in the perfusion channel, which corresponds to the ${ }_{493}$ applied EGF ${ }^{647} /$ Fluorescein concentration.

\subsection{Quantifying EGFR ${ }^{m C i t r i n e}$ phosphorylation in single cells}

To quantify plasma membrane EGFR ${ }^{\text {mCitrine }}$ phosphorylation in live MCF7-EGFR ${ }^{\text {mCitrine }}$ cells, ${ }^{496}$ single cell masks were obtained from the EGFR $^{\text {mCitrine }}$ channel at each time-point using FIJI 497 (https://imagej.net/Fiji). All pixels within the obtained boundary were radially divided into 2498 segments of equal areas (Stanoev et al., 2018), and the outer segment was taken to represent 499 the plasma membrane. For the kymograph analysis, at each time point, the plasma membrane 500 
segment was divided into 4 quadrants in anti-clockwise direction, and each was divided into 501 5 spatial bins (Figure 2A). The fraction of phosphorylated EGFR ${ }^{\text {mCitrine }}$ in each bin, $i$ was 502 estimated as:

$$
E G F R_{p}^{i}(t)=\frac{P T B_{P M}^{i}(t) /\left(P T B_{T}(t)-P T B_{\text {endo }}(t)\right)}{E G F R_{P M}^{i}(t) / E G F R_{T}(t)}
$$

where $P T B_{P M}^{i}(t)$ and $E G F R_{P M}^{i}(t)$ are respectively the PTB $^{\text {mCherry }}$ and EGF $R^{\text {mCitrine }}{ }_{504}$ fluorescence at $i^{\text {th }}$ plasma membrane bin, $P T B_{T}(t)$ and $E G F R_{T}(t)$ - respective total fluores- 505 cence in the whole cell, PTB $B_{\text {endo }}(t)$ - the $\mathrm{PTB}^{m C h e r r y}$ fluorescence on vesicular structures in 506 the cytoplasm. Endosomal structures were identified from the cytosol by intensity thresholding 507 (1.5 s.d. percentile) and $\mathrm{PTB}^{m C h e r r y}$ fluorescence from these structures was subtracted from the 508 $\mathrm{PTB}_{T}(t)$, to correct for the $\mathrm{PTB}^{m C h e r r y}$ fraction bound to the phosphorylated $\mathrm{EGFR}^{\text {mCitrine }}$ on ${ }_{509}$ endosomes.

Temporal profile of the fraction of phosphorylated EGFR ${ }^{\text {mCitrine }}$ on the plasma membrane ${ }_{511}$ was obtained using:

and then normalized as:

$$
E G F R_{p}(t)=\frac{\frac{\sum_{i=1}^{20} P T B_{P M}^{i}(t)}{\left(P T B_{T}(t)-P T B_{\text {endo }}(t)\right)}}{\frac{\sum_{i=1}^{20} E G F R_{P M}^{i}(t)}{\left(E G F R_{T}(t)\right)}}
$$

$$
E G F R_{p}(t)=\frac{\left.E G F R_{p}(t)-<E G F R_{p}>_{t \in[0,5 m i n}\right]}{\max _{t}\left(E G F R_{p}(t)\right)-<E G F R_{p}>_{t \in[0,5 \min ]}}
$$

with $<>$ being the temporal average in the pre-stimulation interval $t \in[0,5 \mathrm{~min}]$. The ${ }_{514}$ fraction of liganded receptor was calculated using:

$$
E G F-E G F R(t)=\frac{E G F_{P M}}{E G F R_{P M}}(t)
$$


To classify single cells into non-activated, activated (polarized EGFR ${ }^{\text {mCitrine }}$ phosphory- 516 lation) and pre-activated (uniformly distributed EGFR ${ }^{m C i t r i n e}$ phosphorylation) upon gradi- 517 ent EGF ${ }^{647}$ stimulation (Figure S2A, B), the following method was applied. To identify pre- 518 activated cells, a Gaussian Mixture $\operatorname{Model}(\mathrm{GMM})$ was fitted to the histogram of $\left(E G F R_{p}^{i}\right)_{t \in\left[0,5 m i \text { rnt }^{\text {19 }}\right.}$ values from all the analysed cells, and the intersection point between the two normal distribu- 520 tions was identified. If more than $30 \%$ of the $\left(E G F R_{p}^{i}\right)_{t \in[0,5 \min ]}$ pixel intensity values for any 521 cell lie above the intersection point, the cell is classified as pre-activated. To distinguish between 522 the non-activated and activated cells in the remaining population, average EGFR ${ }^{m C i t r i n e}$ phos- ${ }_{523}$ phorylation value $\left(E G F R_{p}\right)$ per cell was estimated during the pre-stimulation $(t \in[0,5 \min ]){ }_{524}$ and the stimulation period $(t \in[5 \mathrm{~min}, 65 \mathrm{~min}])\left(<E G F R_{p}>_{t \in[0,65]}\right)$ from the temporal 525 $\mathrm{EGFR}^{\text {mCitrine }}$ phosphorylation profiles. Histogram of the respective $E G F R_{p}$ values was again 526 fitted with a GMM model. All cells with an average $<E G F R_{p}>_{t \in[0,65]}$ value lying below the ${ }_{527}$ intersection point were considered to be non-activated, whereas those above - activated. $\quad 528$

The average of the spatial projection of the fraction of phosphorylated EGFR ${ }^{m C i t r i n e}$ from ${ }_{529}$ single-cell kymographs (Figure S2C) was generated from the 20 (from total of 21 cells) that ${ }_{530}$ were polarized in the direction of the $\mathrm{EGF}^{647}$ gradient. For each cell, a temporal average of ${ }_{531}$ $E G F R_{p}$ per bin was calculated for the duration of the gradient $(t \in[5 \mathrm{~min}, 65 \mathrm{~min}])$ and the ${ }_{532}$ bin with the maximal EGFR $R_{p}$ value was translated to $\pi$. The profiles were then smoothened ${ }_{533}$ using a rolling average with a window of 7 bins. The resulting profiles were then averaged over 534 all cells and mean \pm s.d. is shown.

\subsection{Estimating memory duration in EGFR ${ }^{\text {mCitrine }}$ phosphorylation polar- ${ }_{536}$ ization

The duration of memory in EGFR ${ }^{m \text { Citrine }}$ phosphorylation polarization in single cells was esti- $\quad{ }^{538}$ mated from the temporal profile of the fraction of plasma membrane area with high EGFR ${ }^{m C i t r i n e}{ }_{539}$ 
phosphorylation during and after gradient removal (Figures 2D,E). For this, the single-cell ky- 540 mographs were normalized to a maximal value of 1 using

$$
\operatorname{EGFR}_{p}^{i}(t)=\frac{\left.\operatorname{EGFR}_{p}^{i}(t)-<E G F R_{p}>_{t \in[0,5 m i n}\right]}{\left.\max _{t}\left(E G F R_{p}(t)\right)-<E G F R_{p}>_{t \in[0,5 m i n}\right]}
$$

yielding the value of phosphorylated EGFR ${ }^{m C i t r i n e}$ per bin $i$ per time point $t$. Using the mean of ${ }_{542}$ $E G F R_{p}+s . d$. over the whole experiment duration as a threshold, all $E G F R_{p}^{i}(t)$ lying above ${ }_{543}$ the threshold were taken to constitute the area of polarized EGFR ${ }^{\text {mCitrine }}$ phosphorylation. To ${ }_{544}$ account for different bin sizes, at each timepoint, the area of all bins with $E G F R_{p}$ above the ${ }_{545}$ threshold was summed and divided by the respective total cell area, yielding the temporal evo- 546 lution of the fraction of polarized cell area (FPA) (Figure 2D). The end of the memory duration ${ }_{547}$ per cell was identified as the time point at which FPA $A_{\text {per-cell }}<\left(F P A_{\text {average }}-\right.$ s.d. $)$ in $3 \quad 548$ consecutive time points.

\subsection{Quantifying morphological changes in response to EGF ${ }^{647}$ in exper- 550 iments and simulations

Morphological changes of polarized cells were quantified using the solidity (Figure 2H) of each 552 cell at each time point and the directed protrusive area towards and away from the gradient 553 (Figure $1 \mathrm{G}, \mathrm{H} ; 2 \mathrm{G}$ ). The solidity $\sigma$ is the ratio between the cell's area $A_{\text {cell }}$ and the area of the ${ }_{554}$ convex hull $A_{\text {convex }}\left(\sigma=\frac{A_{\text {cell }}}{A_{\text {convex }}}\right)$. The memory duration in cell morphology was calculated 555 from the single-cell solidity profiles, and corresponds to the time-point at which the solidity is 556 below mean-s.d. estimated during gradient presence. The directed cell protrusion area was esti- 557 mated by comparing single cell masks at two consecutive time points. To reduce noise effects, 558 the masks were first subjected to a 2D Gaussian filtering using the filters.gaussian function $\quad 559$ from the scipy python package. Protrusions were considered if the area change was greater 560 than 10 pixels or $1.2 \mu m^{2}$ per time point. The front and the back of the cells were determined by 561 
identifying an axis that runs perpendicular to the gradient and through the cell nucleus of the ini- 562 tial time point. The directed cell protrusion area was then obtained using $\frac{A_{\text {prot, front }}}{A_{\text {front }}}-\frac{A_{\text {prot }, \text { back }}}{A_{\text {back }}} . \quad 563$ The final profiles of directed protrusive area were smoothed using 1D Gaussian filtering with 564 the filters.gaussian_filter $1 d$ function from the scipy python package. For the equivalent 565 quantification from the simulations, the same procedures were applied without an area thresh- 566 old. The memory duration was estimated as the time point at which the directed protrusive area 567 crosses zero after the gradient removal.

\subsection{Quantification of single-cell migration and duration of memory in ${ }_{569}$ migration

Single cell migration trajectories were extracted using Trackmate (Tinevez et al., 2017) in 571 Fiji (Schindelin et al., 2012) using Hoechst 33342 / transmission channel. From the positional 572 information ( $\mathrm{x}$ and y coordinates) of individual cell tracks, quantities such as Motility, Direc- 573 tionality and $\cos \theta$ were extracted using custom made Python code (Python Software Founda- 574 tion, versions 3.7.3, https://www.python.org/). Directionality was calculated as displacement 575 over total distance and statistical significance was tested using two-sided Welch's t-test. To 576 quantify the memory duration in directed single-cell migration, the Kernel Density Estimate 577 $(\mathrm{KDE})$ from $\cos \theta$ quantification in the continuous absence of $\mathrm{EGF}^{647}$ (uniform case, between ${ }^{578}$ 250 min-300 min) was compared with windowed $\operatorname{KDE}$ (5 time points moving window) from 579 the gradient migration profile, using two sided Kolmogorov-Smirnov test. 580

To quantify the motility patterns of MCF10A cells in absence, uniform or gradient $E G F^{647}{ }_{581}$ stimulation, we fitted the experimentally obtained single cell migration trajectories using modi- 582 fied Ornstein-Uhlenbeck process (mOU) (Uhlenbeck and Ornstein, 1930) that is defined by the ${ }_{583}$ Langevin equation for the velocity vector $\nu$ : 


$$
\frac{d \nu(\mathbf{t})}{d t}=-\frac{1}{\tau} \cdot \nu(\mathbf{t})+\frac{\sqrt{2 D}}{\tau} \cdot(\xi(t)+b(t))
$$

where $\xi(t)$ represents a white noise component, D is a diffusion coefficient characteristic 585 of a Brownian motion, $\tau$ is the persistence time and $b(t)$ models the contribution of the time- 586 dependent bias. The experimental data was fitted to obtain values of $\mathrm{D}$ and $\tau$. In order to 587 estimate D, Mean Square Displacement (MSD) was calculated from the single cell tracks using 588 $M S D(t)=\left\langle\left|\mathbf{x}_{i}(t)-\mathbf{x}_{i}(0)\right|^{2}\right\rangle$, where $\mathbf{x}_{i}(t)$ is the tracked position of $i$-th cell in the 2D ${ }_{589}$ plane, $<>$ is the average across all single cell tracks, and $|$.$| is the Euclidean distance (D. et al., 590$ 2005). To estimate $\mathrm{D}$, the obtained MSD profile was fitted with a linear function $(=4 D t) . \quad 591$ Goodness of Fit for the different experimental conditions: 0ng/ml EGF ${ }^{647}, R^{2}=0.975$; for 592 uniform $20 \mathrm{ng} / \mathrm{ml} \mathrm{EGF}^{647}$ stimulation, $R^{2}=0.995$. In order to estimate $\tau$, Velocity Auto- ${ }_{593}$ Correlation Function $V A C F(t)=\left\langle\nu_{i}(t) \cdot \nu_{i}(0)\right\rangle$, where $\nu_{i}(t)$ is the measured velocity 594 of $i$-th cell at time $\mathrm{t}$, was fitted with a mono exponential function $\left(=\phi_{0} \cdot e^{\frac{-t}{\tau}}\right)$. Goodness of 595 Fit : for 0ng/ml EGF ${ }^{647}$ case - Standard Error Of Estimate $S E O E=0.0261$; for uniform 596 $20 \mathrm{ng} / \mathrm{ml} \mathrm{EGF}^{647}$ stimulation case, $S E O E=0.0570$. Fitted values: for 0ng/ml EGF ${ }^{647}$ case, ${ }_{597}$ $\tau=11.105, D=0.425 ;$ for uniform $20 \mathrm{ng} / \mathrm{ml} \mathrm{EGF}^{647}$ stimulation case, $\tau=38.143, D=2.207 ; \quad 598$ bias $b(t)=0.134$.

\subsection{Reconstructing state-space trajectories from temporal EGFR ${ }^{m C i t r i n e} \quad 600$ phosphorylation profiles

The state-space reconstruction in Figures $2 \mathrm{~F}$ and $3 \mathrm{G}$ was performed using the method of time- 602 delay. For a time series of a scalar variable, a vector $x\left(t_{i}\right), i=1, . . N$ in state-space in time $t_{i} \quad{ }_{603}$ can be constructed as following 


$$
\mathbf{X}\left(t_{i}\right)=\left[x\left(t_{i}\right), x\left(t_{i}+\tau\right), . ., x\left(t_{i}+(m-1) \tau\right)\right]
$$

where $i=1$ to $N-(m-1) \tau, \tau$ is the embedding delay, $m$ - is a dimension of reconstructed 605 space (embedding dimension). Following the embedding theorems by Takens (Takens, 1980) 606 and Sauer et al. (Sauer et al., 1991), if the sequence $X\left(t_{i}\right)$ consists of scalar measurements of 607 the state of a dynamical system, then under certain genericity assumptions, the time delay em- 608 bedding provides a one-to-one image of the original set, provided $m$ is large enough. The em- ${ }^{609}$ bedding delay was identified using the timeLag function (based on autocorrelation), the embed- 610 ding dimension using the estimateEmbeddingDims function (based on the nearest-neighbours 611 method), and the state-space reconstruction using the buildTakens function, all from the nonlin- 612 earTseries package in R (https://cran.r-project.org/web/packages/nonlinearTseries/index.html). ${ }_{613}$ Before state-space reconstructions, time series were smoothened using the Savitzky-Golay filter 614 function in Python. For Figure $2 \mathrm{~F}, \tau=26, d_{e}=3$; for Figure $3 \mathrm{G}, \tau=50, d_{e}=3$. 615 


\section{References and Notes}

Albrecht, E. and Petty, H. R. (1998). Cellular memory: Neutrophil orientation reverses during temporally decreasing chemoattractant concentrations. Proceedings of the National Academy of Sciences 95, 5039-5044.

Atkinson, R. and Shiffrin, R. (1968). Human memory: A proposed system and its control processes. Physiology of Learning and Motivation 2, 89-195.

Baumdick, M., Bruggemann, Y., Schmick, M., Xouri, G., Sabet, O., Davis, L., Chin, J. W. and Bastiaens, P. I. (2015). EGF-dependent re-routing of vesicular recycling switches spontaneous phosphorylation suppression to EGFR signaling. eLife 4, e12223.

Becherer, P., Morozov, A. N. and van Saarloos, W. (2009). Probing a subcritical instability with an amplitude expansion: An exploration of how far one can get. Physica D: Nonlinear Phenomena 238, 1827-1840.

Beta, C., Amselem, G. and Bodenschatz, E. (2008). A bistable mechanism for directional sensing. New J of Phys 10, 083015.

Bjorkelund, H., Gedda, L., Malmqvist, M. and Andersson, K. (2012). Resolving the EGFEGFR interaction characteristics through a multiple-temperature, multiple-inhibitor, real-time interaction analysis approach. Mol. Clin. Oncol. 1, 343-352.

Brueggemann, Y., Karajannis, L. S., Stanoev, A., Stallaert, W. and Bastiaens, P. I. H. (2021). Growth factor-dependent ErbB vesicular dynamics couple receptor signaling to spatially and functionally distinct Erk pools. Science Signaling 14, eabd9943.

Chiasson-MacKenize, C. and McClatchey, A. I. (2018). EGFR-induced cytoskeletal changes drive complex cell behavior: The tip of the iceberg. Science Signaling 11, eaas9473. 
D., S., Mosler, S., Hagedorn, P., Larsen, N. and Flyvbjerg, H. (2005). Cell Motility as Persistent Random Motion: Theories from Experiments. Biophysical J. 89, 912-931.

Foxman, E. F., Kunkel, E. J. and Butcher, E. C. (1999). Integrating Conflicting Chemotactic Signals: The Role of Memory in Leukocyte Navigation. Journal of Cell Biology 147, 577-588.

Goryachev, A. B. and Pokhilko, A. V. (2008). Dynamics of CDC42 network embodies a Turingtype mechanism of yeast cell polarity. FEBS Lett. 582, 1437-1443.

Halatek, J. and Frey, E. (2018). Rethinking pattern formation in reaction-diffusion systems. Nature Physics 14, 507-514.

Hochreiter, S. and Schmidhuber, J. (1997). Long short-term memory. Neural Comp. 9, 17351780.

Jilkine, A. and Edelstein-Keshet, L. (2011). A Comparison of Mathematical Models for Polarization of Single Eukaryotic Cells in Response to Guided Cues. PLOS Computational Biology $7,1-15$.

Koseska, A., Volkov, E. and Kurths, J. (2013). Transition from amplitude to oscillation death via Turing bifurcation. Phys Rev Lett 111, 024103.

Levchenko, A. and Iglesias, P. A. (2002). Models of eukaryotic gradient sensing: application to chemotaxis of amoebae and neutrophils. Biophysical J 82, 50-63.

Levine, H., Kessler, D. A. and Rappel, W.-J. (2002). Directional sensing in eukaryotic chemotaxis: a balanced inactivation model. PNAS 103, 9761-9766.

Maass, W., Natschlager, T. and Markram, H. (2000). Real-time computing without stable states: a new framework for neural computation based on perturbations. Neural Comp. 14, 2531-2560. 
Mori, Y., A., J. and Edelstein-Keshet, L. (2008). Wave-pinning and cell polarity from a bistable reaction-diffusion model. Biophysical J. 94, 3684-3697.

Offterdinger, M., Georget, V., Girod, A. and Bastiaens, Philippe, I. (2004). Imaging phosphorylation dynamics of the epidermal growth factor receptor. J Biol Chem. 279, 36972-81.

Paquin-Lefebvre, F., Xu, B., DiPietro, K. L., Lindsay, A. E. and Jilkine, A. (2020). Pattern formation in a coupled membrane-bulk reaction-diffusion model for intracellular polarization and oscillations. Journal of Theoretical Biology 497, 110242.

Parent, C. A. and Devreotes, P. N. (1999). A cell's sense of direction. Science 284, 765-770.

Prentice-Mott, H. V., Meroz, Y., Carlson, A., Levine, M. A., Davidson, M. W., Irimia, D., Charras, G. T., Mahadevan, L. and Shah, J. V. (2016). Directional memory arises from longlived cytoskeletal asymmetries in polarized chemotactic cells. Proceedings of the National Academy of Sciences 113, 1267-1272.

Reynolds, A., Tischer, C., Verveer, P., Rocks, O. and Bastiaens, P. (2003). EGFR activation coupled to inhibition of tyrosine phosphatases causes lateral signal propagation. Nat Cell Biol $5,447-453$.

Ridley, A. and Hall, A. (1992). The small GTP-binding protein rho regulates the assembly of focal adhesions and actin stress fibers in response to growth factors. Cell 70, 389-399.

Ridley, A. J., Schwartz, M. A., Burridge, K., Firtel, R. A., Ginsberg, M. H., Borisy, G., Parsons, J. T. and Horwitz, A. R. (2003). Cell Migration: Integrating Signals from Front to Back. Science $302,1704-1709$.

Sauer, T., Yorke, J. and Casdagli, M. (1991). Embedology. J. Stat. Phys. 65, 579. 
Schindelin, J., Arganda-Carreras, I., Frise, E., Kaynig, V., Longair, M., Pietzsch, T., Preibisch, S., Rueden, C., Saalfeld, S., Schmid, B., Tinevez, J.-Y., White, D. J., Hartenstein, V., Eliceiri, K., Tomancak, P. and Cardona, A. (2012). Fiji: an open-source platform for biological-image analysis. Nature Methods 9, 676-682.

Skoge, M., Yue, H., Erickstad, M., Bae, A., Levine, H., Groisman, A., Loomis, W. F. and Rappel, W.-J. (2014). Cellular memory in eukaryotic chemotaxis. Proceedings of the National Academy of Sciences $111,14448-14453$.

Sonntag, M., Ibach, J., Nieto, L., Verveer, P. and Brunsveld, L. (2014). Site-Specific Protection and Dual Labeling of Human Epidermal Growth Factor (hEGF) for Targeting, Imaging, and Cargo Delivery. Chemistry - a European Journal 20, 6019-6026.

Stanoev, A., Mhamane, A., Schuermann, K., Grecco, H., Stallaert, W., Baumdick, M., Brüggemann, Y., Joshi, M., Roda-Navarro, P., Fengler, S., Stockert, R., Roßmannek, L., Luig, J., Koseska, A. and Bastiaens, P. (2018). Interdependence between EGFR and Phosphatases Spatially Established by Vesicular Dynamics Generates a Growth Factor Sensing and Responding Network. Cell Systems 7, 295-309.e11.

Stanoev, A., Nandan, A. P. and Koseska, A. (2020). Organization at criticality enables processing of time-varying signals by receptor networks. Mol Syst Biol 16, e8870.

Strogatz, S. H. (2018). Nonlinear dynamics and chaos: with applications to physics, biology, chemistry, and engineering. CRC Press.

Svensson, C., Medyukhina, A., Belyaev, I., Al-Zaben, N. and Figge, M. (2017). Untangling cell tracks: Quantifying cell migration by time lapse image data analysis. Cytometry Part A, J. of Quantitative Cell Science 93A, 357-370. 
Takens, F. (1980). "Detecting strange attractors in turbulence", in ”Dynamical systems and turbulence”, Editors David Rand and Lai-Sang Young. Springer Nature.

Tinevez, J.-Y., Perry, N., Schindelin, J., Hoopes, G. M., Reynolds, G. D., Laplantine, E., Bednarek, S. Y., Shorte, S. L. and Eliceiri, K. W. (2017). TrackMate: An open and extensible platform for single-particle tracking. Methods 115, 80-90.

Trong, P. K., Nicola, E. M., Goehring, N. W., Kumar, K. V. and Grill, S. W. (2014). Parameterspace topology of models for cell polarity. New J of Physics 16, 065009.

Uhlenbeck, G. and Ornstein, L. (1930). On the theory of Brownian motion. Phys. Rev. 36, 823-841.

Xiong, Y., Huang, C.-H., Iglesias, P. and Devreotes, P. N. (2010). Cells navigate with a localexcitation, global-inhibition-biased excitable network. PNAS 107, 17079-17086.

Yang, L., Effler, J. C., Kutscher, B. L., Sullivan, S. E., Robinson, D. N. and Iglesias, P. A. (2008). Modeling cellular deformations using the level set formalism. BMC Syst Biol 2, 1-16. 